\title{
Taxes, Capital Structure Choices, and Equity Value
}

\author{
Mara Faccio and Jin Xu*
}

\begin{abstract}
We use a multitude of tax reforms across the Organisation for Economic Co-Operation and Development (OECD) countries as natural experiments to estimate the market value of the tax benefits of debt financing. We report time-series evidence that tax reforms are followed by large changes in the value of corporate equity. However, the impact of tax reforms is greatly mitigated by the presence of leverage. The value of debt tax savings is greater among top taxpayers, among highly profitable firms, and in countries where tax laws are more strongly enforced. Importantly, the value of debt tax savings is in line with the benchmark implied by a traditional approach.
\end{abstract}

\section{Introduction}

The Modigliani and Miller (1963) model of capital structure has formed the basis of finance pedagogy for over half a century. Despite this, the actual magnitude of the tax benefits of debt financing has long been the topic of considerable theoretical and empirical dispute. ${ }^{1}$ According to Fama (2011), currently, "[T]he big open challenge in corporate finance is to produce evidence on how taxes affect market values and thus optimal financing decisions" (p. 8).

*Faccio, mfaccio@purdue.edu, Purdue University Krannert School of Management, Asian Bureau of Finance and Economic Research (ABFER), European Corporate Governance Institute (ECGI), and National Bureau of Economic Research (NBER); Xu (corresponding author), xujin@vt.edu, Virginia Polytechnic Institute and State University Pamplin College of Business. We thank an anonymous referee, Najah Attig, John Graham, Kate Holland, Yeejin Jang, E. Han Kim, Paul Malatesta (the editor), Vikram Nanda, Bill O'Brien, Abraham Ravid, Bohui Zhang, and seminar participants at the 2014 NBER Summer Institute, the 2014 Conference on Financial Economics and Accounting at Georgia State University, the 2015 American Finance Association meetings, the 2016 Tsinghua Finance Workshop, Aalto University, the University of Chicago, the Hanken School of Economics, Pennsylvania State University, Purdue University, Saint Mary's University (Halifax), the University of Arkansas, the University of Illinois at Chicago, and Virginia Tech for comments.

${ }^{1}$ Empirical work includes Masulis (1980), McConnell and Schlarbaum (1981), Bradley, Jarrell, and Kim (1984), Fama and French (1998), and Graham (2000). Theoretical studies include Miller (1977), DeAngelo and Masulis (1980), and Green and Hollifield (2003), to cite a few. Extensions of those studies include Dotan and Ravid (1985) and Dammon and Senbet (1988). The latter two studies investigate how taxes, leverage, and investment interact. 
In this paper, we respond to this challenge. We exploit tax reforms affecting statutory corporate or personal tax rates in the Organisation for Economic Co-Operation and Development (OECD) countries to directly estimate the market value attached to the tax benefits of debt financing. The sample comprises over 300 reforms affecting corporate and/or personal tax rates across 29 OECD countries and spanning more than 3 decades. $^{2}$ A major benefit of our approach (which differentiates us from most prior studies) is that we isolate shocks directly affecting the tax benefits of debt financing. This allows us to attach a clear tax interpretation to our results.

We document that the impact of tax reforms on value is mitigated by the presence of leverage. For example, in the presence of leverage, the positive value impact of a reform reducing the corporate tax rate is, in part, offset by a decline in the present value of debt tax shields. Importantly, we find that the mitigating effect of leverage on the effect of tax changes on value is economically large. In support of a tax interpretation of our results, we document that debt tax shields are more valuable for firms subject to a higher effective tax rate and for more profitable firms. We further document that debt tax shields are less valuable in countries with high levels of tax evasion and in countries whose laws enable more stealing by insiders. In contrast, debt tax shields are more valuable in countries with low levels of tax evasion and in countries where stealing by insiders is relatively more difficult.

Our main tests use change regression specifications. Those regressions include an array of variables to control for the impact of tax reforms on capital structure decisions, investments, factor demand and growth, and, ultimately, future expected cash flows. We take several additional steps to mitigate endogeneity concerns.

First, we include country-year fixed effects (FEs) to control for unobserved shocks that might coincide with tax reforms and similarly affect all firms in a given country at a given point in time. Thus, in our models, identification comes from the differential response to a given tax reform as a function of a firm's leverage ratio.

Second, we include interactions between the changes in the tax rates and each of our control variables. These interactions mitigate the concern that tax changes might affect firm value through a channel other than leverage, such as firm-level investment, change in investment opportunities, and growth. We also add interactions between the changes in the personal tax rates and the firm's earnings to account for the possibility that the reforms might affect value through the discounting of future cash flows.

Third, we show that the results are robust to using a narrow event window in an event study setting. We focus on tax reforms introduced as surprises and examine value changes around the dates of the reform news announcements that we were able to identify. By narrowing the window around which value changes are measured, we are able to filter out a number of possible non-tax-related events.

\footnotetext{
${ }^{2}$ Some of these reforms occur at the local level, in which case the tax rate changes tend to be small. Although we include all tax changes in the main analyses, we specifically focus on large tax reforms in some later tests.
} 
This mitigates the possibility that the results might be due to events (other than the tax reforms) that affect firms through a leverage channel. We also show that the results are not driven by the specific methodology employed (e.g., they are robust to using a propensity score matching approach), nor do they appear to be driven by possible biases in the estimation process.

While we study a simple question in this paper, our results are especially important to the corporate finance literature. Perhaps of greatest importance is the economic magnitude of the benefits associated with debt tax shields. We use both the propensity score matching test and the event study to assess the magnitude of the tax benefits of debt financing. Although the event study perhaps captures less than the full effect of the tax reforms, it is less likely to be contaminated by confounding events. We calculate the theoretical benchmark for the difference in the value change around a tax reform between high- and low-leverage firms following a traditional approach. In this approach, we assume that the gross tax benefit of debt equals the product of debt amount and the corporate tax rate. We find our empirical estimate of the tax benefits of debt from the propensity score matching results to be statistically indistinguishable from the theoretical benchmark. By comparison, the estimate from the event study equals approximately $70 \%$ of the theoretical benchmark. We, thus, conclude that the tax benefits of $\$ 1$ of debt financing approximates $\$ 1 \times T_{C}$, where $T_{C}$ is the corporate tax rate.

Our paper relates to recent studies that estimate the benefits of debt financing. In a seminal paper, Graham (2000) simulates the benefit functions of interest tax deductibility and employs those to estimate the tax savings associated with each incremental dollar of interest payments. He estimates a tax benefit of debt financing of approximately $7 \%-10 \%$ of firm value, depending on whether personal taxes are considered. By comparison, he notes that the "traditional approach" of measuring tax benefits as the product of the amount of debt and the corporate tax rate yields an estimate equal to $13 \%$ of firm value. ${ }^{3}$ Using a different approach based on shocks to the tax rates induced by tax reforms, we find the tax benefits of debt financing to be sizeable. Specifically, we estimate the tax benefits of debt financing to be consistent with the benchmark implied by the traditional approach.

Our paper is closely related to a study by Doidge and Dyck (2015), who focus on a reform that eliminated the tax benefits given to Canadian income trusts. (Prior to the reform, Canadian income trusts could avoid the payment of corporate taxes.) These authors document that income trusts using tax shields were affected less by the reform. Unlike our study, theirs focuses on a single reform affecting only Canadian income trusts. It is, therefore, not obvious whether their results generalize to other tax reforms.

\footnotetext{
${ }^{3}$ van Binsbergen, Graham, and Yang (2010) focus on firms that appear to be optimally levered to estimate the benefit and cost of debt functions for individual firms. They estimate the net (of debt costs) benefit of debt to be on average around $3.5 \%$ of firm value. Using a different approach based on an extension of Modigliani and Miller (1958), Korteweg (2010) estimates the net benefits of debt to be $5.5 \%$ of firm value for the median firm. Thus, as summarized by Graham and Leary (2011), the more recent evidence suggests that capital structure choices appear to have only a modest impact on firm value for many firms.
} 
Our paper is also related to studies of how corporations change their capital structure in response to tax reforms (Campello (2001), Desai, Foley, and Hines (2004), Fan, Titman, and Twite (2012), Givoly, Hahn, Ofer, and Sarig (1992), Gordon and MacKie-Mason (1991), Rajan and Zingales (1995), and Twite (2001)). In two recent studies, Faccio and Xu (2015) and Heider and Ljungqvist (2015) provide evidence that firms substantially rebalance their capital structure in response to tax reforms both internationally and across U.S. states. However, Bargeron, Denis, and Lehn (2018), who look at the introduction of corporate and individual taxes in the United States in the early 1900s, find little evidence of taxes as a primary determinant of capital structure choices. In this paper, we investigate whether there appears to be a value to adjusting leverage in response to tax reforms.

The rest of the paper is organized as follows: Section II describes the empirical approach and the data. Section III presents the main results. Section IV documents that the tax benefits of debt financing are more valuable for top taxpayers. Section V presents the event study results, and Section VI assesses the robustness of the results using a propensity score matching methodology. Section VII discusses the economic magnitude of the results. Section VIII presents a number of robustness tests. Those include the following: Allowing for differences in the allequity cost of capital across industries, investigating the results reform by reform, accounting for expected future cash flows, accounting for taxes on capital gains, and allowing for the business cycle to differently affect firms with different levels of leverage. Section IX concludes.

\section{Identification Strategy and Data}

\section{A. Identification Strategy}

The starting point of our empirical approach is the observation that the market value of a levered firm $\left(V_{L}\right)$ can be decomposed into i) the present value of the tax gains (or losses) from leverage, ii) the present value of other benefits and costs of debt, and iii) the market value of the unlevered firm. The tax gains (or losses) from leverage reflect the deductibility of interest payments from taxable income at the corporate level and the taxation of income from debt and/or equity at the personal level. In Miller (1977), for example, the tax gains (or losses) from a perpetual amount of debt, $D$, are equal to $D \times\left[1-\left(\left(1-T_{C}\right) \times\left(1-T_{E}\right)\right) /\left(1-T_{D}\right)\right]$. $\left(T_{C}\right.$ is the corporate (income) tax rate, $T_{E}$ is the personal tax rate on income from equity, and $T_{D}$ is the personal tax rate on interest income.) Other benefits of debt include managerial commitment to operating efficiency and monitoring by lenders. The other costs include financial distress costs, agency costs, and debt overhang. ${ }^{4}$ The market value of the unlevered firm is equal to the present value of its unlevered expected cash flows $(\mathrm{E}(\mathrm{OCF}))$, net of the theoretical corporate taxes on those cash flows, discounted at the all-equity cost of capital.

\footnotetext{
${ }^{4}$ Notice that the other benefits and/or costs of debt change around a tax reform if i) firms rebalance their capital structure and/or ii) tax reforms overlap with other reforms (e.g., bankruptcy or governance reforms) that affect value through a leverage channel. Focusing on a narrow event window later in the paper enables us to filter out these contaminating events.
} 
Within this framework, a change in the corporate tax rate $\left(\delta T_{C}\right)$ is expected to impact firm value because it affects i) the value of the tax gains from leverage and ii) the (after-tax) value of the unlevered firm. A change in the personal tax rate on income from equity $\left(\delta T_{E}\right)$ or debt $\left(\delta T_{D}\right)$ is also expected to affect the value of the tax gains from leverage. The expected impact of tax rate changes on value varies across firms as a function of the level of outstanding debt. For example, ceteris paribus, while a corporate tax increase results in a drop in firm value, this effect is expected to be less pronounced for highly levered firms, as those firms are able to shield more income from corporate taxes. ${ }^{5}$

With this in mind, the backbones of the basic regression models that we test are the following:

$$
\begin{aligned}
\frac{\delta V_{L}}{A}= & \alpha+\beta_{1} \times \delta T_{C} \times \frac{D}{A}+\gamma \times \delta T_{C} \times \frac{\mathrm{E}(\mathrm{OCF})}{A}+\varepsilon \\
\frac{\delta V_{L}}{A}= & \alpha+\beta_{1} \times \delta T_{C} \times \frac{D}{A}+\beta_{2} \times \delta T_{D} \times \frac{D}{A}+\beta_{3} \times \delta T_{E} \times \frac{D}{A} \\
& +\gamma \times \delta T_{C} \times \frac{\mathrm{E}(\mathrm{OCF})}{A}+\varepsilon .
\end{aligned}
$$

The only difference between the two models is whether personal taxes are considered. The following are expected to hold: i) $\beta_{1}>0$, ii) $\beta_{2}<0$, iii) $\beta_{3}>0$, and iv) $\gamma<0$. Using $\delta V_{L}$ as the dependent variable is potentially problematic, as the results would likely be dominated by the largest firms in the sample. To deal with this problem, we scale both the dependent and the independent variables by lagged total book assets, $A$.

Following equations (1) and (2), we estimate the market value of interest tax shields using change regression specifications. In those specifications, annual changes in value are regressed on contemporaneous changes in tax rates, interacted with the degree of leverage at the beginning of the year, along with several firm-level control variables. The inclusion of firm-level controls accounts for the fact that we rely on a relatively simple valuation model. While in our specifications we use (current) earnings before interest and taxes (divided by lagged total assets) to proxy for expected cash flows, we recognize that tax reforms might affect future cash flows through a growth channel. For example, following a taxreducing reform, firms might have greater incentives to invest; as a consequence, their earnings would subsequently increase. Therefore, we use an array of variables (e.g., changes in earnings; changes in property, plant, and equipment; and changes in $R \& D$ expenses) to capture the impact of reforms on future investments and expected cash flows. (These controls are described in Section II.B.)

Further, to the extent that the tax benefits of debt financing are indeed valuable, we would expect leverage to change in response to tax reforms.

\footnotetext{
${ }^{5}$ Consider two firms with the same operating income of $\$ 100$. (Assume for simplicity that $T_{E}=T_{D}$.) Firm A is unlevered. Firm B, which is levered, pays annual interest of $\$ 100$, which is tax deductible. If the corporate tax rate is $10 \%$, firm A pays $\$ 10$ (10\% of its taxable income of $\$ 100)$ in corporate income taxes, while firm B pays $\$ 0$. If the corporate tax rate increases to $50 \%$, ceteris paribus, firm A pays income taxes of $\$ 50$, while firm B pays no income taxes. As such, the value of the unlevered firm should drop more than the value of a highly levered firm.
} 
Indeed, Faccio and Xu (2015) and Heider and Ljungqvist (2015) provide evidence that firms rebalance their capital structure following tax reforms. (Presumably, firms change their capital structure up to the point where the new marginal tax benefits of debt financing equal the marginal costs.) Therefore, in all models, we control for changes in leverage that occur (possibly) in response to tax reforms.

Our identification strategy relies on the following assumptions: i) No event other than the tax rate changes that we focus on generates the different changes in value that we observe across firms with different leverage ratios; ii) the change in value indeed occurs through a leverage channel. We undertake several steps to minimize concerns with this identification strategy.

First, we include country-year FEs to control for any country-level observable and unobservable shocks that might correlate with the tax reforms and similarly affect the value of all firms in a given country. Second, we include interactions between the changes in tax rates and each of our control variables. These interactions control for the possibility that the tax reforms might affect different firms differently. More importantly, these interactions control for the possibility that tax changes might affect firms through a channel other than leverage, such as growth, change in investment opportunities, or the discounting of future cash flows. For example, if a change in personal tax rates affects the after-tax cost of equity or debt, this may affect the value of the firm separately from any tax shield effects. Therefore, to investigate whether the tax reforms affect firm value through the discounting of future cash flows, in some specifications, we interact each firm's expected cash flows with the changes in personal tax rates. We discuss these channels in more detail in Section III.

Third, we show later in the paper that the results are also robust to using a narrow event window in an event study setting. By narrowing the window around which value changes are measured, we filter out a number of possible non-taxrelated events and further mitigate the possibility that the results might be due to events (other than the tax reforms) that might affect firms through a leverage channel. A benefit of this approach is that it does not require us to compile a comprehensive list of all possible value-relevant events that could contaminate our results. (Compiling such a list would be a formidable task.)

In all specifications, standard errors are double clustered at the country-year and at the firm level. Clustering at the country-year level accounts for the correlation in the responses of different firms to each given tax reform. Clustering at the firm-level accounts for serial correlation.

\section{B. Data}

Tax data come from Faccio and Xu (2015). The sample covers 29 OECD countries and 29 years spanning from 1981 through 2009. CORPORATE TAX_CHANGE $\left(\delta T_{C}\right)$ is the annual change in the top marginal statutory corporate income tax rate. This variable includes national and regional corporate income taxes. INTEREST_TAX_CHANGE $\left(\delta T_{D}\right)$ is the annual change in the highest marginal tax rate applied to residents' personal interest income from corporate bonds. DIVIDEND_TAX_CHANGE $\left(\delta T_{E}\right)$ is the annual change in the net top statutory tax rate on dividend income to be paid at the shareholder level. 
This variable takes into account any gross-up provisions and reliefs available at the shareholder level. ${ }^{6}$

The sample contains 190 changes in corporate tax rates, 103 changes in the personal tax rates on interest income, and 205 changes in the personal tax rates on dividend income. These reforms span 307 unique country-years, leaving a sample of 252 country-years in which no tax rate change occurs. The sample includes 137 tax rate changes equal to or greater than 500 basis points. These larger reforms are fairly evenly distributed across countries and through time. Overall, there appears to be a sufficient degree of identifying variation in tax rate changes, both across and within countries and through time.

Firm-level data are taken from Worldscope and Datastream. Throughout the paper, financial companies (Standard Industrial Classification (SIC) codes: 6***) and regulated utilities (SIC codes: 49**) are excluded from the analyses. With the exception of those in Section $\mathrm{V}$, all analyses use the change in the market value of equity minus any change in the book value of equity $\left(d \mathrm{VNI}_{t} / A_{t-1}\right)$ as a measure of the change in firm value $\left(\delta V_{L} / A\right)$.

Following Fama and French (1998), $d \mathrm{VNI}_{t} / A_{t-1}$ is computed as the annual change in the market value of equity minus the annual change in the book value of equity (which captures financing activities), all divided by lagged book assets. We recognize that this variable accurately captures overall changes in firm value only if the market value of debt does not change. We also recognize that changes in the personal tax rates on interest payments will presumably affect the market valuation of debt, which our empirical proxy cannot capture. Our choice is by necessity. In particular, we are unable to control for changes in the market value of outstanding debt, as most firm debt is not publicly traded.

The analyses in Section V use the 5-day CUMULATIVE_STOCK_RETURN as the dependent variable. The 5-day CUMULATIVE_STOCK_RETURN is the sum of daily stock returns during the $(-2,+2)$ day interval surrounding a corporate tax reduction news event, multiplied by the firm's market value of equity and divided by the firm's book value of total assets.

LEVERAGE $_{t-1}(D / A)$ is lagged interest-bearing debt divided by lagged total assets. $E_{t} / A_{t-1}(\mathrm{E}(\mathrm{OCF}) / A)$ is earnings before interest and taxes divided by lagged total assets. $\ln \left(\text { SALES }_{t} \text { ) is the natural } \log \text { of (net) sales. (M_B) }\right)_{t-1}$ is the lagged market-to-book ratio, defined as total assets minus book equity plus market equity, all divided by total assets. $d E_{t} / A_{t-1}$ is the change in earnings before interest and taxes divided by lagged total assets. ${ }^{7} d \mathrm{NPPE}_{t} / A_{t-1}$ is the change in net property, plant, and equipment divided by lagged total assets. $d \mathrm{RD}_{t} / A_{t-1}$ is the annual change in research and development expenditures divided by lagged total assets. $d \mathrm{DEBT}_{t} / A_{t-1}$ is the difference between the level of interest-bearing debt divided by lagged total assets. CYCLICAL_INDUSTRY is an indicator that takes the value of 1 for any 2-digit SIC industry whose performance is perceived to be positively related to the overall business cycle, and 0 for "countercyclical"

\footnotetext{
${ }^{6}$ Capital gains taxes, which are available only for a subset of countries and years, are analyzed in Section VIII.D.

${ }^{7}$ The inclusion of this variable allows us to indirectly control for changes in non-debt tax shields, such as depreciation or any other tax-deductible cost.
} 
industries. As in Faccio and $\mathrm{Xu}$ (2015), countercyclical industries are identified based on keyword searches. ${ }^{8}$

The sample includes 203,676 firm-year observations. As shown in Table 1, the average corporate tax rate is $38.41 \%$, the average personal tax rate on interest income is $33.51 \%$, and the average personal tax rate on dividend income is $26.34 \%$. During our sample period, tax rates decline through time, on average.

TABLE 1

Summary Statistics

In Table 1, CORPORATE TAX (CORPORATE_TAX_CHANGE) is the (annual change in the) top marginal statutory corporate income tax rate. INTEREST_TAX (INTEREST_TAX_CHANGE) is the (annual change in the) highest marginal tax rate applied to residents' personal interest income from corporate bonds. DIVIDEND_TAX (DIVIDEND_TAX_CHANGE) is the (annual change in the) net top statutory tax rate on dividend income to be paid at the shareholder level. $d \mathrm{VNI}_{t} / A_{t-1}$ is the change in the market value of equity, minus any change in the book value of equity, all divided by lagged book assets. $E_{t} / A_{t-1}$ is earnings before interest and taxes divided by lagged total assets. In(SALES $)$ is the natural log of net sales. $\left(\mathrm{M}_{-} B\right)_{t-1}$ is the lagged market-to-book ratio, defined as total assets minus book equity plus market equity, all divided by total assets. $d E_{t} / A_{t-1}$ is the change in earnings before interest and taxes divided by lagged total assets. NPPE $t / A_{t}$ is net property, plant, and equipment, all divided by total assets. $d \mathrm{NPPE}_{t} / A_{t-1}$ is the change in NPPE divided by lagged total assets. $d \mathrm{RD}_{t} / A_{t-1}$ is the annual change in research and development expenditures divided by lagged total assets. Leverage is interest-bearing debt divided by total assets. CYCLICAL_INDUSTRY is an indicator that takes the value of 1 for any 2-digit Standard Industrial Classification $(\mathrm{SIC})$ industry whose performance is perceived to be positively related to the overall business cycle, and 0 for countercyclical industries. $d \mathrm{DEBT} t / A_{t-1}$ is the difference between the level of interest-bearing debt at the end of year $t$ and the level of interest-bearing debt at the end of year $t-1$, all scaled by total assets as of the end of year $t-1$.

\begin{tabular}{|c|c|c|c|c|}
\hline Variable & $\begin{array}{l}\text { No. of } \\
\text { Obs. }\end{array}$ & Mean & Median & $\begin{array}{l}\text { Std. } \\
\text { Dev. }\end{array}$ \\
\hline CORPORATE_TAX & 203,676 & 0.3841 & 0.3930 & 0.0668 \\
\hline INTEREST_TAX & 203,241 & 0.3351 & 0.3500 & 0.1064 \\
\hline DIVIDEND_TAX & 203,676 & 0.2634 & 0.2720 & 0.1087 \\
\hline CORPORATE_TAX_CHANGE & 203,676 & -0.0050 & 0.0000 & 0.0174 \\
\hline INTEREST_TAX_CHANGE & 203,241 & -0.0031 & 0.0000 & 0.0264 \\
\hline DIVIDEND_TAX_CHANGE & 203,676 & -0.0100 & 0.0000 & 0.0557 \\
\hline$d \mathrm{VNI}_{t} / A_{t-1}$ & 203,676 & 0.0908 & 0.0058 & 1.6761 \\
\hline$E_{t} / A_{t-1}$ & 203,676 & 0.0137 & 0.0621 & 0.2779 \\
\hline $\ln \left(\right.$ SALES $\left._{t}\right)$ & 203,676 & 12.1754 & 12.2885 & 2.3590 \\
\hline$\left(\mathrm{M} \_\mathrm{B}\right)_{t-1}$ & 203,676 & 1.9724 & 1.2839 & 2.5969 \\
\hline$d \overline{E_{t}} / A_{t-1}$ & 203,676 & 0.0154 & 0.0070 & 0.2548 \\
\hline$d \mathrm{NPPE}_{t} / A_{t-1}$ & 203,676 & -0.0001 & -0.0010 & 0.0628 \\
\hline$d \mathrm{RD}_{t} / A_{t-1}$ & 203,676 & 0.0021 & 0.0000 & 0.0204 \\
\hline LEVERAGE $_{t-1}$ & 203,676 & 0.2392 & 0.2047 & 0.2122 \\
\hline CYCLICAL_INDUSTRY & 203,676 & 0.7784 & 1.0000 & 0.4153 \\
\hline$d \mathrm{DEBT} / / A_{t-1}$ & 203,676 & 0.0321 & 0.0000 & 0.1720 \\
\hline
\end{tabular}

\section{Main Results}

The main regression results are reported in Table 2 . In regression 1 , we test a simple model with taxation only at the corporate level. Regression 2 is a test of the more general valuation model with taxation at the corporate and personal levels. The results are consistent with the predictions in Section II.A. In particular, the impact of an increase in corporate tax rates on the equity value of the firms affected by the tax reform is less pronounced as leverage increases, as $\beta_{1}$

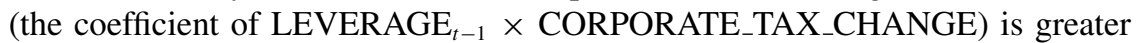
than 0 . At the same time, the benefits associated with the deductibility of interest

${ }^{8}$ Those comprise agricultural production crops (2-digit SIC code: 01); agriculture production livestock and animal specialties (02); agricultural services (07); fishing, hunting, and trapping (09); food and kindred products (20); tobacco products (21); chemicals and allied products (28); electric, gas, and sanitary services (49); wholesale trade-non-durable goods (51); food stores (54); health services (80); legal services (81); and educational services (82). 
TABLE 2

\section{Leverage and the Impact of Tax Reforms on Value}

In Table 2, the dependent variable $d \mathrm{VNI}_{t} / A_{t-1}$ is the change in the market value of equity, minus any change in the book value of equity, all divided by lagged book assets. All other variables are defined in Table 1. "Corporate tax reform years" are years in which the corporate tax rate changes. "Corporate or personal tax reform years" are years with a change in at least one of the corporate tax rate, the personal interest tax rate, or the personal dividend tax rate. All regression models include country-year fixed effects. $t$-statistics based on standard errors adjusted for 2-way clustering (i.e., at the country-year and at the firm level) are shown in the parentheses below the coefficient estimates. *, **, and *** indicate significance at the $10 \%, 5 \%$, and $1 \%$ levels, respectively. The tax change variables drop out of the models naturally due to the inclusion of country-year fixed effects.

\begin{tabular}{|c|c|c|c|c|c|c|}
\hline \multirow[b]{3}{*}{ Variable } & \multirow[b]{3}{*}{1} & \multirow[b]{3}{*}{2} & \multirow[b]{3}{*}{3} & \multicolumn{3}{|c|}{ Subsample Including Only: } \\
\hline & & & & \multirow{2}{*}{$\begin{array}{c}\text { Corporate } \\
\text { Tax Reform } \\
\text { Years } \\
4 \\
\end{array}$} & \multicolumn{2}{|c|}{$\begin{array}{l}\text { Corporate or Personal } \\
\text { Tax Reform Years }\end{array}$} \\
\hline & & & & & 5 & 6 \\
\hline $\begin{array}{l}\text { LEVERAGE }_{t-1} \\
\times \text { CORPORATE_TAX_CHANGE }\end{array}$ & $\begin{array}{l}5.150^{*} \\
(1.83)\end{array}$ & $\begin{array}{l}8.202^{* \star} \\
(2.31)\end{array}$ & $\begin{array}{l}7.270^{\star \star} \\
(2.10)\end{array}$ & $\begin{array}{l}8.729^{* *} \\
(1.90)\end{array}$ & $\begin{array}{l}9.390^{\star \star} \\
(2.03)\end{array}$ & $\begin{array}{l}8.606^{*} \\
(1.87)\end{array}$ \\
\hline $\begin{array}{l}\text { LEVERAGE }_{t-1} \\
\quad \times \text { INTEREST_TAX_CHANGE }\end{array}$ & & $\begin{array}{l}-8.831^{\star \star *} \\
(-2.10)\end{array}$ & $\begin{array}{l}-4.842 \\
(-1.48)\end{array}$ & & $\begin{array}{l}-8.725^{\star \star} \\
(-2.16)\end{array}$ & $\begin{array}{l}-4.706 \\
(-1.51)\end{array}$ \\
\hline $\begin{array}{l}\text { LEVERAGE }_{t-1} \\
\quad \times \text { DIVIDEND_TAX_CHANGE }\end{array}$ & & $\begin{array}{l}3.273^{*} \\
(1.65)\end{array}$ & $\begin{array}{l}4.384^{\star \star *} \\
(2.64)\end{array}$ & & $\begin{array}{l}3.713^{*} \\
(1.80)\end{array}$ & $\begin{array}{l}4.853^{* * *} \\
(2.63)\end{array}$ \\
\hline $\begin{array}{l}E_{t} / A_{t-1} \\
\quad \times \text { CORPORATE_TAX_CHANGE }\end{array}$ & $\begin{array}{l}-27.174^{\star *} \\
(-1.99)\end{array}$ & $\begin{array}{l}-24.824^{* \star} \\
(-1.96)\end{array}$ & $\begin{array}{l}-25.651^{\star \star} \\
(-2.00)\end{array}$ & $\begin{array}{l}-26.302^{*} \\
(-1.88)\end{array}$ & $\begin{array}{l}-24.010^{*} \\
(-1.93)\end{array}$ & $\begin{array}{l}-24.225^{\star *} \\
(-1.96)\end{array}$ \\
\hline $\begin{array}{l}E_{t} / A_{t-1} \\
\quad \times \text { INTEREST_TAX_CHANGE }\end{array}$ & & & $\begin{array}{l}6.882 \\
(0.97)\end{array}$ & & & $\begin{array}{c}7.079 \\
(1.01)\end{array}$ \\
\hline $\begin{array}{l}E_{t} / A_{t-1} \\
\quad \times \text { DIVIDEND_TAX_CHANGE }\end{array}$ & & & $\begin{array}{l}4.533 \\
(0.98)\end{array}$ & & & $\begin{array}{l}4.803 \\
(1.03)\end{array}$ \\
\hline $\ln \left(\right.$ SALES $\left._{t}\right)$ & $\begin{array}{l}-0.029^{\star \star \star} \\
(-4.32)\end{array}$ & $\begin{array}{l}-0.025^{\star \star \star} \\
(-4.04)\end{array}$ & $\begin{array}{l}-0.027^{\star \star \star} \\
(-4.36)\end{array}$ & $\begin{array}{l}-0.035^{\star \star \star} \\
(-3.58)\end{array}$ & $\begin{array}{l}-0.031^{\star \star \star} \\
(-4.03)\end{array}$ & $\begin{array}{l}-0.034^{\star \star \star *} \\
(-4.39)\end{array}$ \\
\hline$\left(\mathrm{M} \_\mathrm{B}\right)_{t-1}$ & $\begin{array}{l}-0.086^{\star \star \star} \\
(-2.80)\end{array}$ & $\begin{array}{l}-0.093^{\star \star \star} \\
(-2.93)\end{array}$ & $\begin{array}{l}-0.092^{\star \star \star} \\
(-2.91)\end{array}$ & $\begin{array}{l}-0.087^{\star \star} \\
(-2.35)\end{array}$ & $\begin{array}{l}-0.084^{\star \star} \\
(-2.27)\end{array}$ & $\begin{array}{l}-0.082^{* *} \\
(-2.24)\end{array}$ \\
\hline$d E_{t} / A_{t-1}$ & $\begin{array}{l}0.231^{*} \\
(1.84)\end{array}$ & $\begin{array}{l}0.255^{*} \\
(1.88)\end{array}$ & $\begin{array}{l}0.223^{*} \\
(1.69)\end{array}$ & $\begin{array}{l}0.098 \\
(0.66)\end{array}$ & $\begin{array}{r}0.157 \\
(1.02)\end{array}$ & $\begin{array}{l}0.116 \\
(0.78)\end{array}$ \\
\hline$d \mathrm{NPPE}_{t} / A_{t-1}$ & $\begin{array}{l}-1.817^{\star * *} \\
(-6.63)\end{array}$ & $\begin{array}{l}-1.795^{\star \star \star} \\
(-6.65)\end{array}$ & $\begin{array}{l}-1.794^{\star * *} \\
(-6.64)\end{array}$ & $\begin{array}{l}-2.521^{\star \star *} \\
(-6.37)\end{array}$ & $\begin{array}{l}-2.306^{\star * \star} \\
(-6.95)\end{array}$ & $\begin{array}{l}-2.304^{\star \star \star *} \\
(-6.94)\end{array}$ \\
\hline$d \mathrm{RD}_{t} / A_{t-1}$ & $\begin{array}{l}4.640^{\star \star \star *} \\
(4.52)\end{array}$ & $\begin{array}{l}4.998^{\star \star \star} \\
(4.82)\end{array}$ & $\begin{array}{l}5.001^{\star \star \star} \\
(4.81)\end{array}$ & $\begin{array}{l}4.711^{* \star \star} \\
(3.64)\end{array}$ & $\begin{array}{l}5.400^{\star \star \star} \\
(4.48)\end{array}$ & $\begin{array}{l}5.400^{\star \star \star \star} \\
(4.47)\end{array}$ \\
\hline LEVERAGE $_{t-1}$ & $\begin{array}{l}0.024 \\
(0.24)\end{array}$ & $\begin{array}{l}0.029 \\
(0.26)\end{array}$ & $\begin{array}{l}0.044 \\
(0.41)\end{array}$ & $\begin{array}{c}0.102 \\
(0.64)\end{array}$ & $\begin{array}{l}0.055 \\
(0.37)\end{array}$ & $\begin{array}{l}0.076 \\
(0.52)\end{array}$ \\
\hline$E_{t} / A_{t-1}$ & $\begin{array}{l}-0.768^{\star \star *} \\
(-3.80)\end{array}$ & $\begin{array}{l}-0.761^{\star \star \star} \\
(-3.88)\end{array}$ & $\begin{array}{l}-0.696^{\star \star \star} \\
(-3.53)\end{array}$ & $\begin{array}{l}-0.753^{\star \star \star} \\
(-3.13)\end{array}$ & $\begin{array}{l}-0.710^{\star \star \star} \\
(-3.25)\end{array}$ & $\begin{array}{l}-0.625^{\star * \star} \\
(-2.86)\end{array}$ \\
\hline$d \mathrm{DEBT}_{t} / A_{t-1}$ & $\begin{array}{l}0.633^{* * *} \\
(3.69)\end{array}$ & $\begin{array}{l}0.636^{\star \star \star} \\
(3.78)\end{array}$ & $\begin{array}{l}0.637^{\star \star *} \\
(3.78)\end{array}$ & $\begin{array}{l}0.697^{\star \star \star \star} \\
(2.69)\end{array}$ & $\begin{array}{l}0.637^{\star \star \star} \\
(3.07)\end{array}$ & $\begin{array}{l}0.640^{* * *} \\
(3.09)\end{array}$ \\
\hline $\begin{array}{l}d \mathrm{DEBT}_{t} / A_{t-1} \\
\quad \times \text { CORPORATE_TAX_CHANGE }\end{array}$ & $\begin{array}{l}7.275 \\
(0.67)\end{array}$ & $\begin{array}{l}8.708 \\
(0.88)\end{array}$ & $\begin{array}{r}7.211 \\
(0.59)\end{array}$ & $\begin{array}{l}9.968 \\
(0.85)\end{array}$ & $\begin{array}{l}8.912 \\
(0.74)\end{array}$ & $\begin{array}{r}7.427 \\
(0.61)\end{array}$ \\
\hline $\begin{array}{l}d \mathrm{DEBT} T_{t} / A_{t-1} \\
\times \text { INTEREST_TAX_CHANGE }\end{array}$ & & $\begin{array}{l}-6.853 \\
(-0.59)\end{array}$ & $\begin{array}{l}-4.070 \\
(-0.33)\end{array}$ & & $\begin{array}{l}-6.794 \\
(-0.61)\end{array}$ & $\begin{array}{l}-3.986 \\
(-0.34)\end{array}$ \\
\hline $\begin{array}{l}d \mathrm{DEBT}_{t} / A_{t-1} \\
\quad \times \mathrm{DIVIIDEND}_{-} \text {TAX_CHANGE }\end{array}$ & & $\begin{array}{l}4.620 \\
(0.88)\end{array}$ & $\begin{array}{c}4.842 \\
(0.92)\end{array}$ & & $\begin{array}{l}4.708 \\
(0.95)\end{array}$ & $\begin{array}{l}4.960 \\
(0.98)\end{array}$ \\
\hline $\begin{array}{l}\text { LEVERAGE }_{t-1} \\
\times \text { CORPORATE_TAX_CHANGE } \\
\times \text { CYCLICAL_INDUSTRY }\end{array}$ & $\begin{array}{c}2.988 \\
(1.52)\end{array}$ & $\begin{array}{c}2.670 \\
(1.26)\end{array}$ & $\begin{array}{c}3.127 \\
(1.39)\end{array}$ & $\begin{array}{c}2.771 \\
(1.08)\end{array}$ & $\begin{array}{c}2.468 \\
(1.01)\end{array}$ & $\begin{array}{c}2.937 \\
(1.12)\end{array}$ \\
\hline $\begin{array}{l}\text { LEVERAGE }_{t-1} \\
\times \text { INTEREST_TAX_CHANGE } \\
\times \text { CYCLICAL_INDUSTRY }\end{array}$ & & $\begin{array}{c}1.190 \\
(1.09)\end{array}$ & $\begin{array}{l}-0.025 \\
(-0.01)\end{array}$ & & $\begin{array}{l}1.223^{\star \star \star} \\
(3.60)\end{array}$ & $\begin{array}{c}0.004 \\
(0.00)\end{array}$ \\
\hline $\begin{array}{l}\text { LEVERAGE }_{t-1} \\
\times \text { DIVIDEND_TAX_CHANGE } \\
\times \text { CYCLICAL_INDUSTRY }\end{array}$ & & $\begin{array}{l}-0.577 \\
(-0.93)\end{array}$ & $\begin{array}{l}-0.589 \\
(-0.71)\end{array}$ & & $\begin{array}{l}-0.667 \\
(-1.07)\end{array}$ & $\begin{array}{l}-0.662 \\
(-0.79)\end{array}$ \\
\hline $\begin{array}{l}E_{t} / A_{t-1} \\
\times \text { CORPORATE_TAX_CHANGE } \\
\quad \times \text { CYCLICAL_INDUSTRY }\end{array}$ & $\begin{array}{l}-10.683 \\
(-0.99)\end{array}$ & $\begin{array}{l}-11.797 \\
(-1.06)\end{array}$ & $\begin{array}{l}-14.302 \\
(-1.34)\end{array}$ & $\begin{array}{c}-13.545 \\
(-1.23)\end{array}$ & $\begin{array}{l}-13.885 \\
(-1.25)\end{array}$ & $\begin{array}{l}-16.305 \\
(-1.52)\end{array}$ \\
\hline
\end{tabular}


TABLE 2 (continued)

\section{Leverage and the Impact of Tax Reforms on Value}

\begin{tabular}{|c|c|c|c|c|c|c|}
\hline \multirow[b]{3}{*}{ Variable } & \multirow[b]{3}{*}{1} & \multirow[b]{3}{*}{2} & \multirow[b]{3}{*}{3} & \multicolumn{3}{|c|}{ Subsample Including Only: } \\
\hline & & & & \multirow{2}{*}{$\begin{array}{c}\text { Corporate } \\
\text { Tax Reform } \\
\text { Years } \\
4 \\
\end{array}$} & \multicolumn{2}{|c|}{$\begin{array}{c}\text { Corporate or Personal } \\
\text { Tax Reform Years }\end{array}$} \\
\hline & & & & & 5 & 6 \\
\hline $\begin{array}{l}E_{t} / A_{t-1} \\
\times \text { INTEREST_TAX_CHANGE } \\
\quad \times \text { CYCLICAL_INDUSTRY }\end{array}$ & & & $\begin{array}{l}4.638 \\
(0.70)\end{array}$ & & & $\begin{array}{l}4.569 \\
(0.71)\end{array}$ \\
\hline $\begin{array}{l}E_{t} / A_{t-1} \\
\quad \times \text { DIVIDEND_TAX_CHANGE } \\
\quad \times \text { CYCLICAL_INDUSTRY }\end{array}$ & & & $\begin{array}{l}-0.568 \\
(-0.15)\end{array}$ & & & $\begin{array}{l}-0.819 \\
(-0.22)\end{array}$ \\
\hline $\begin{array}{l}d \text { DEBT } t / A_{t-1} \\
\times \text { CORPORATE_TAX_CHANGE } \\
\times \text { CYCLICAL_INDUSTRY }\end{array}$ & $\begin{array}{r}3.980 \\
(0.34)\end{array}$ & $\begin{array}{r}4.280 \\
(0.33)\end{array}$ & $\begin{array}{r}4.075 \\
(0.32)\end{array}$ & $\begin{array}{l}-0.072 \\
(-0.01)\end{array}$ & $\begin{array}{l}3.964 \\
(0.31)\end{array}$ & $\begin{array}{r}3.749 \\
(0.31)\end{array}$ \\
\hline $\begin{array}{l}d \mathrm{dDEBT} / A_{t-1} \\
\times \text { INTEREST_TAX_CHANGE } \\
\times \text { CYCLICAL_INDUSTRY }\end{array}$ & & $\begin{array}{l}-1.101 \\
(-0.09)\end{array}$ & $\begin{array}{l}-1.343 \\
(-0.12)\end{array}$ & & $\begin{array}{l}-1.272 \\
(-0.11)\end{array}$ & $\begin{array}{l}-1.563 \\
(-0.14)\end{array}$ \\
\hline $\begin{array}{l}d \text { DEBT } t / A_{t-1} \\
\times \text { DIVIDEND_TAX_CHANGE } \\
\times \text { CYCLICAL_INDUSTRY }\end{array}$ & & $\begin{array}{l}2.733 \\
(0.41)\end{array}$ & $\begin{array}{r}2.707 \\
(0.41)\end{array}$ & & $\begin{array}{l}2.584 \\
(0.40)\end{array}$ & $\begin{array}{l}2.528 \\
(0.40)\end{array}$ \\
\hline $\begin{array}{l}\text { LEVERAGE }_{t-1} \\
\quad \times \text { CYCLICAL_INDUSTRY }\end{array}$ & $\begin{array}{l}0.205^{\star *} \\
(1.99)\end{array}$ & $\begin{array}{l}0.202^{\star \star} \\
(1.97)\end{array}$ & $\begin{array}{l}0.201^{\star \star} \\
(1.99)\end{array}$ & $\begin{array}{l}0.267^{*} \\
(1.68)\end{array}$ & $\begin{array}{r}0.220 \\
(1.59)\end{array}$ & $\begin{array}{r}0.219 \\
(1.62)\end{array}$ \\
\hline $\begin{array}{l}E_{t} / A_{t-1} \\
\quad \times \text { CYCLICAL_INDUSTRY }\end{array}$ & $\begin{array}{l}0.471^{\star *} \\
(2.35)\end{array}$ & $\begin{array}{l}0.476^{\star \star} \\
(2.36)\end{array}$ & $\begin{array}{l}0.485^{\star \star *} \\
(2.28)\end{array}$ & $\begin{array}{l}0.437^{*} \\
(1.79)\end{array}$ & $\begin{array}{l}0.428^{\star} \\
(1.90)\end{array}$ & $\begin{array}{l}0.438^{*} \\
(1.82)\end{array}$ \\
\hline $\begin{array}{l}d \mathrm{DEBT}_{t} / A_{t-1} \\
\quad \times \text { CYCLICAL_INDUSTRY }\end{array}$ & $\begin{array}{l}-0.022 \\
(-0.12)\end{array}$ & $\begin{array}{l}-0.017 \\
(-0.09)\end{array}$ & $\begin{array}{l}-0.021 \\
(-0.11)\end{array}$ & $\begin{array}{l}-0.123 \\
(-0.45)\end{array}$ & $\begin{array}{l}-0.040 \\
(-0.18)\end{array}$ & $\begin{array}{l}-0.045 \\
(-0.20)\end{array}$ \\
\hline CYCLICAL_INDUSTRY & $\begin{array}{l}-0.104^{\star \star} \\
(-2.54)\end{array}$ & $\begin{array}{l}-0.106^{\star \star \star} \\
(-2.61)\end{array}$ & $\begin{array}{l}-0.106^{\star * \star} \\
(-2.66)\end{array}$ & $\begin{array}{l}-0.136^{\star \star} \\
(-1.96)\end{array}$ & $\begin{array}{l}-0.118^{\star \star} \\
(-2.03)\end{array}$ & $\begin{array}{l}-0.120^{* \star} \\
(-2.08)\end{array}$ \\
\hline $\begin{array}{l}\operatorname{In}\left(\mathrm{SALES}_{t}\right) \\
\quad \times \text { CORPORATE_TAX_CHANGE }\end{array}$ & $\begin{array}{l}-0.375^{\star *} \\
(-2.81)\end{array}$ & $\begin{array}{l}-0.576^{\star \star \star} \\
(-2.83)\end{array}$ & $\begin{array}{l}-0.484^{* * *} \\
(-2.92)\end{array}$ & $\begin{array}{l}-0.465^{\star \star \star} \\
(-2.98)\end{array}$ & $\begin{array}{l}-0.657^{\star \star \star} \\
(-3.28)\end{array}$ & $\begin{array}{l}-0.574^{* * *} \\
(-3.40)\end{array}$ \\
\hline $\begin{array}{l}\left(\mathrm{M} \_B\right)_{t-1} \\
\times \text { CORPORATE_TAX_CHANGE }\end{array}$ & $\begin{array}{c}1.834 \\
(1.31)\end{array}$ & $\begin{array}{r}1.251 \\
(0.76)\end{array}$ & $\begin{array}{r}1.305 \\
(0.81)\end{array}$ & $\begin{array}{c}1.839 \\
(1.26)\end{array}$ & $\begin{array}{r}1.475 \\
(0.88)\end{array}$ & $\begin{array}{c}1.538 \\
(0.95)\end{array}$ \\
\hline $\begin{array}{l}d E_{t} / A_{t-1} \\
\quad \times \text { CORPORATE_TAX_CHANGE }\end{array}$ & $\begin{array}{l}-0.572 \\
(-0.13)\end{array}$ & $\begin{array}{l}-3.152 \\
(-0.64)\end{array}$ & $\begin{array}{l}-3.595 \\
(-0.75)\end{array}$ & $\begin{array}{l}-3.237 \\
(-0.71)\end{array}$ & $\begin{array}{l}-4.887 \\
(-0.97)\end{array}$ & $\begin{array}{l}-5.451 \\
(-1.13)\end{array}$ \\
\hline $\begin{array}{l}d \mathrm{NPPE}_{t} / A_{t-1} \\
\times \text { CORPORATE_TAX_CHANGE }\end{array}$ & $\begin{array}{l}-9.095^{\star} \\
(-1.88)\end{array}$ & $\begin{array}{l}-6.686 \\
(-1.22)\end{array}$ & $\begin{array}{l}-7.231 \\
(-1.30)\end{array}$ & $\begin{array}{l}-20.050^{\star \star \star} \\
(-3.10)\end{array}$ & $\begin{array}{l}-14.318^{\star \star} \\
(-2.45)\end{array}$ & $\begin{array}{l}-14.834^{* *} \\
(-2.51)\end{array}$ \\
\hline $\begin{array}{l}d \mathrm{RD}_{t} / A_{t-1} \\
\quad \times \text { CORPORATE_TAX_CHANGE }\end{array}$ & $\begin{array}{c}45.756 \\
(1.47)\end{array}$ & $\begin{array}{c}45.471 \\
(1.23)\end{array}$ & $\begin{array}{l}47.855 \\
(1.32)\end{array}$ & $\begin{array}{c}47.829 \\
(1.52)\end{array}$ & $\begin{array}{c}53.474 \\
(1.44)\end{array}$ & $\begin{array}{r}55.847 \\
(1.53)\end{array}$ \\
\hline $\begin{array}{l}\ln \left(\text { SALES }_{t}\right) \\
\quad \times \text { INTEREST_TAX_CHANGE }\end{array}$ & & $\begin{array}{r}0.343 \\
(1.31)\end{array}$ & $\begin{array}{c}0.116 \\
(1.07)\end{array}$ & & $\begin{array}{r}0.340 \\
(1.34)\end{array}$ & $\begin{array}{c}0.109 \\
(1.14)\end{array}$ \\
\hline $\begin{array}{l}\left(\mathrm{M} \_\mathrm{B}\right)_{t-1} \\
\quad \times \text { INTEREST_TAX_CHANGE }\end{array}$ & & $\begin{array}{l}0.666 \\
(0.74)\end{array}$ & $\begin{array}{l}0.570 \\
(0.64)\end{array}$ & & $\begin{array}{r}0.661 \\
(0.74)\end{array}$ & $\begin{array}{r}0.561 \\
(0.64)\end{array}$ \\
\hline $\begin{array}{l}d E_{t} / A_{t-1} \\
\quad \times \text { INTEREST_TAX_CHANGE }\end{array}$ & & $\begin{array}{r}5.951 \\
(1.45)\end{array}$ & $\begin{array}{r}2.480 \\
(0.73)\end{array}$ & & $\begin{array}{c}5.683 \\
(1.40)\end{array}$ & $\begin{array}{l}2.133 \\
(0.62)\end{array}$ \\
\hline $\begin{array}{l}d \mathrm{NPPE}_{t} / A_{t-1} \\
\times \text { INTEREST_TAX_CHANGE }\end{array}$ & & $\begin{array}{l}-5.217 \\
(-1.32)\end{array}$ & $\begin{array}{l}-4.918 \\
(-1.27)\end{array}$ & & $\begin{array}{l}-5.179 \\
(-1.46)\end{array}$ & $\begin{array}{l}-4.894 \\
(-1.42)\end{array}$ \\
\hline $\begin{array}{l}d \mathrm{RD}_{t} / A_{t-1} \\
\quad \times \text { INTEREST_TAX_CHANGE }\end{array}$ & & $\begin{array}{r}7.730 \\
(0.30)\end{array}$ & $\begin{array}{l}-1.313 \\
(-0.05)\end{array}$ & & $\begin{array}{l}6.373 \\
(0.25)\end{array}$ & $\begin{array}{l}-2.642 \\
(-0.10)\end{array}$ \\
\hline $\begin{array}{l}\ln \left(\mathrm{SALES}_{t}\right) \\
\quad \times \text { DIVIDEND_TAX_CHANGE }\end{array}$ & & $\begin{array}{l}0.437^{\star} \\
(1.82)\end{array}$ & $\begin{array}{l}0.289^{\star * *} \\
(3.48)\end{array}$ & & $\begin{array}{l}0.413^{*} \\
(1.76)\end{array}$ & $\begin{array}{l}0.263^{\star \star \star} \\
(3.50)\end{array}$ \\
\hline $\begin{array}{l}\left(\mathrm{M} \_\mathrm{B}\right)_{t-1} \\
\quad \times \text { DIVIDEND_TAX_CHANGE }\end{array}$ & & $\begin{array}{l}-0.877 \\
(-1.53)\end{array}$ & $\begin{array}{l}-0.647 \\
(-1.16)\end{array}$ & & $\begin{array}{l}-0.809 \\
(-1.39)\end{array}$ & $\begin{array}{l}-0.575 \\
(-1.01)\end{array}$ \\
\hline $\begin{array}{l}d E_{t} / A_{t-1} \\
\quad \times \text { DIVIDEND_TAX_CHANGE }\end{array}$ & & $\begin{array}{r}1.430 \\
(0.59)\end{array}$ & $\begin{array}{c}0.332 \\
(0.17)\end{array}$ & & $\begin{array}{r}0.850 \\
(0.35)\end{array}$ & $\begin{array}{l}-0.287 \\
(-0.15)\end{array}$ \\
\hline $\begin{array}{l}d \mathrm{NPPE}_{t} / A_{t-1} \\
\quad \times \text { DIVIDEND_TAX_CHANGE }\end{array}$ & & $\begin{array}{r}2.970 \\
(0.70)\end{array}$ & $\begin{array}{l}2.848 \\
(0.70)\end{array}$ & & $\begin{array}{l}1.288 \\
(0.34)\end{array}$ & $\begin{array}{c}1.156 \\
(0.32)\end{array}$ \\
\hline $\begin{array}{l}d \mathrm{RD}_{t} / A_{t-1} \\
\quad \times \text { DIVIDEND_TAX_CHANGE }\end{array}$ & & $\begin{array}{l}35.025 \\
(1.46)\end{array}$ & $\begin{array}{c}30.195 \\
(1.24)\end{array}$ & & $\begin{array}{c}37.408 \\
(1.56)\end{array}$ & $\begin{array}{c}32.654 \\
(1.35)\end{array}$ \\
\hline Country-year fixed effects & Yes & Yes & Yes & Yes & Yes & Yes \\
\hline $\begin{array}{l}\text { No. of obs. } \\
\text { Adj. } R^{2}\end{array}$ & $\begin{array}{r}203,676 \\
0.078 \\
\end{array}$ & $\begin{array}{r}203,241 \\
0.082 \\
\end{array}$ & $\begin{array}{r}203,241 \\
0.083 \\
\end{array}$ & $\begin{array}{r}113,617 \\
0.071 \\
\end{array}$ & $\begin{array}{r}139,788 \\
0.072 \\
\end{array}$ & $\begin{array}{r}139,788 \\
0.074 \\
\end{array}$ \\
\hline
\end{tabular}


payments at the corporate level are at least partly offset by the disadvantage associated with the taxation of interest payments at the personal level (so that $\beta_{2}<0$ ). The taxation of dividend income at the personal level, however, further increases the overall tax benefits of debt $\left(\beta_{3}>0\right)$. While personal taxes on dividend income matter at the margin, it is perhaps not surprising that they do not appear to matter as much as corporate tax rates or personal tax rates on interest income. One explanation relates to the relatively small (and declining) number of dividendpaying firms studied during our sample period (Fama and French (2001), Denis and Osobov (2008)).

Further, $\gamma$ (the coefficient of $E_{t} / A_{t-1} \times$ CORPORATE_TAX_CHANGE) is negative. This indicates that, following an increase in corporate tax rates, the equity value of more profitable firms drops by a larger amount. (Note that, because of the inclusion of country-year FEs, the tax change variables drop out of the models.)

Importantly, in this paper, we contribute to the literature by addressing concerns about various possible omitted variables. One such variable of special interest is taxation (of dividends and interest) at the personal level. The evidence from regression 2 is consistent with the idea that personal taxes affect the market value of debt tax shields. Further, a comparison of columns 1 and 2 of Table 2 shows

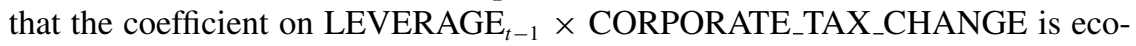
nomically larger when personal tax changes are controlled for. Because of the inclusion of country-year FEs, the results cannot be explained by omitted shocks that might affect all firms in a given country-year in the same way. In principle, another possible concern with the results is that the tax reforms might affect firms through a channel other than leverage. The inclusion of interactions between the changes in tax rates and each of our control variables mitigates this possibility.

Among these alternative stories, one concern is that tax reforms might affect value through a "growth channel." More specifically, firms with different growth profiles may respond to tax reforms differently. To the extent that leverage is correlated with growth, our result may merely reflect this alternative channel. To minimize this concern, we include interactions between our firm-level growth variables $\left(d E_{t} / A_{t-1}, d \mathrm{NPPE}_{t} / A_{t-1}\right.$, and $\left.d \mathrm{RD}_{t} / A_{t-1}\right)$ and each tax change variable. Across the six regression specifications shown in Table 2, this produces a total of 42 interactions among these three firm-level growth variables and the tax change variables. Only four of these interactions are statistically significant, while the other 38 interaction terms are not statistically significant at conventional levels. Overall, this appears to provide little support for a growth-based story.

Another possibility is that the tax reforms affect firms' investment opportunities. For example, following a reduction in the corporate tax rate, some of the projects that previously had negative net present values will have positive net present values. The growth variables discussed in the previous paragraph account for short-term changes in property, plant, and equipment as well as changes in earnings that will result from undertaking these additional projects.

We also investigate the possibility that the results might instead reflect the different response of firms (with different leverage ratios) to economic shocks that might coincide with tax reforms. If this channel is behind our results, we would expect the coefficient of the LEVERAGE $\times$ TAX_CHANGE interactions 
to be larger for firms in industries that are sensitive to economic cycles (cyclical industries). For this purpose, we focus on triple interactions: LEVERAGE $\times$ TAX_CHANGE $\times$ CYCLICAL_INDUSTRY. An important benefit of the cyclical industry indicator is that it accounts for future growth as well. Across the six regression specifications in Table 2, only one of the 14 triple interactions is significant at the $10 \%$ level or better, providing little support for the hypothesis that our results reflect a different response of firms to economic shocks. Of the remaining interaction terms, none of the interactions between the market-to-book ratio and the tax change variables is statistically significant, while a number of the interaction terms between firm size and tax changes are significant. Importantly, the LEVERAGE $\times$ TAX_CHANGE interactions that are the focus of this paper are statistically significant after controlling for this battery of interactions.

Another possibility is that tax reforms affect value through the discounting of future cash flows. Specifically, if a change in personal tax rates affects the after-tax cost of equity or debt, this would affect the value of the firm separate from any tax shield effects. To investigate this possibility, in regression 3 of Table 2, we interact personal tax rate changes with expected cash flows. The interaction enables us to test whether personal tax rate changes affect firm value through another channel, that is, the discounting of future cash flows. We find that neither of the interactions between $\mathrm{E}(\mathrm{OCF}) / A$ and the personal tax changes is significant. This provides little support to the idea that tax reforms affect equity values through the discounting of future cash flows.

In regressions 4-6 of Table 2, we assess the robustness of our results to changes in the sample composition. In particular, regression 4 includes only country-years in which a reform affecting corporate tax rates occurs. The co-

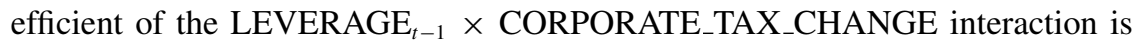
positive and significant in this specification as well. Regressions 5 and 6 include only country-years in which either a reform affecting corporate tax rates or a reform affecting personal tax rates occurs. In general, we find that the results for the subsample of years in which tax reforms occur are very similar to those in regressions $1-3$.

\section{Additional Evidence: Top Taxpayers}

Tax reforms should have different effects on firms as a function of each firm's marginal tax rate. Therefore, in this section, we investigate whether the value of debt tax shields is greater i) for firms subject to a higher marginal tax rate and ii) in countries in which stealing (including tax evasion) is more difficult. Such evidence would further strengthen our tax interpretation of the leverage results.

The regressions in Tables 3 and 4 are run for the full sample of firms, that is, both high and low effective tax rate firms, both high and low ROA firms, and both dividend payers and non-payers. The evidence in Table 3 indicates that the firms that should respond more to tax changes do exhibit a higher (equity) value response to the tax reforms. In regression 1, we use a firm's EFFECTIVE_TAX_RATE, measured as the ratio of taxes paid over pretax income, as a proxy for the firm's marginal tax rate. We use this variable to construct an indicator, HIGH_EFFECTIVE_TAX_RATE, which equals 1 if the effective tax rate is above its median value for the country and year, and 0 otherwise. 
TABLE 3

Leverage and the Impact of Tax Reforms on Value: Top Taxpayers

In Table 3, the dependent variable $d \mathrm{VNI}_{t} / A_{t-1}$ is the change in the market value of equity, minus any change in the book value of equity, all divided by lagged book assets. HIGH EFFECTIVE TAX RATE equals 1 if the effective tax rate is above its median value for the country and year, and 0 otherwise. In regression 1 , the HIGH_EFFECTIVE_TAX_RATE is defined as taxes paid over pretax income. In regression 2, the HIGH_EFFECTIVE_TAX_RATE is the sum of taxes paid in years $-2,-1$, and 0 , divided by the sum of pretax income in the same 3 years. HIGH_ROA equals 1 if a firm's ROA is above its median value for the country and year, and 0 otherwise. DIVIDEND_PAYER equals 1 if the firm pays cash dividends in a given year, and 0 otherwise. All other variables are defined in Table 1. All regression models include country-year fixed effects. The regression models also include the interactions between each of the control variables and corporate and personal tax changes, although their coefficients are omitted for brevity. $t$-statistics based on standard errors adjusted for 2-way clustering (i.e., at the country-year and at the firm level) are shown in the parentheses below the coefficient estimates. ${ }^{*},{ }^{* *}$, and ${ }^{* * *}$ indicate significance at the $10 \%, 5 \%$, and $1 \%$ levels, respectively.

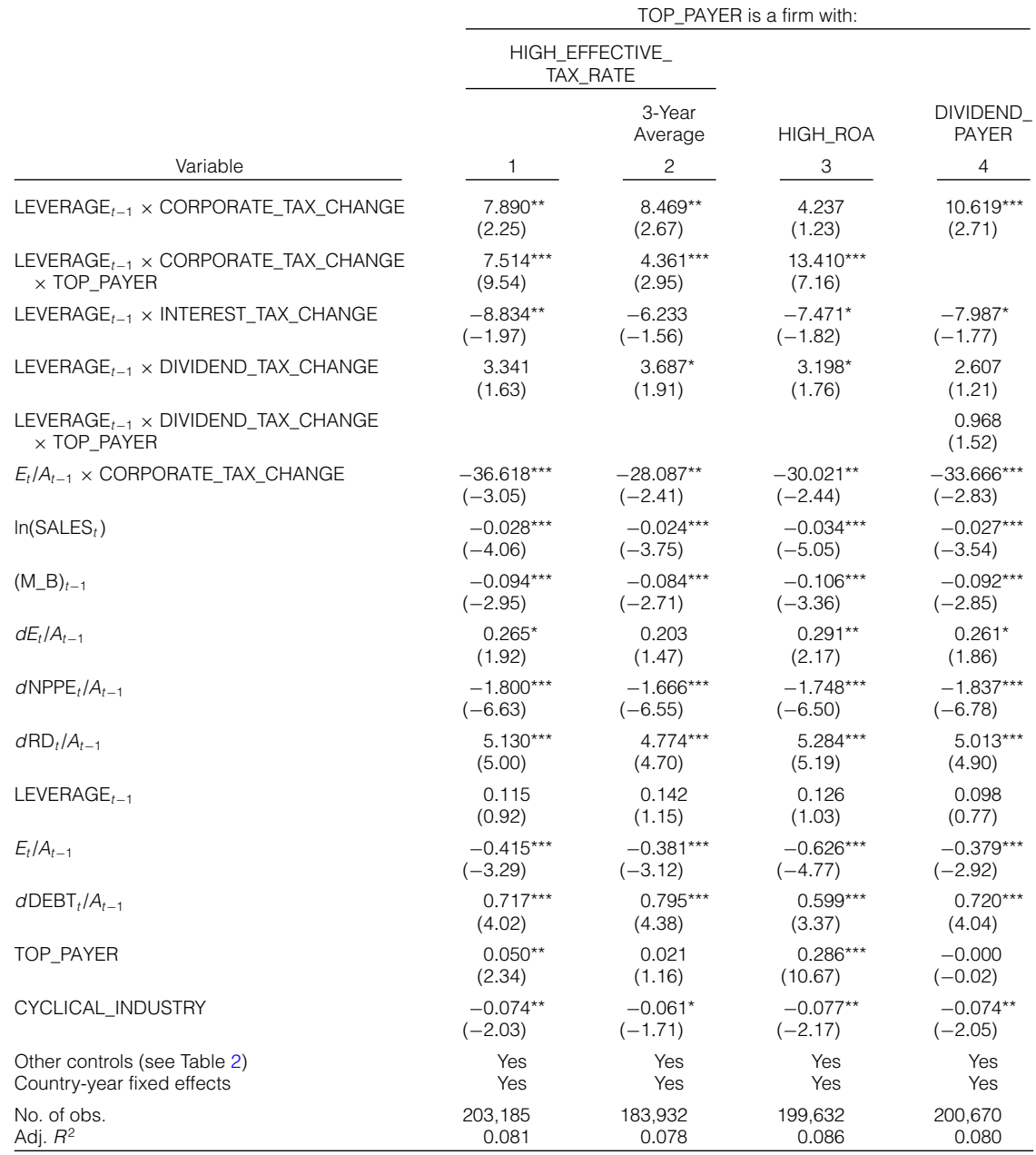

Thus, in regression 1 , the coefficient of the LEVERAGE L $_{t-1} \times$ CORPORATE $_{-}$ TAX_CHANGE interaction reflects the importance of a corporate tax change (i.e., the differential effect of corporate tax changes on value depending on financial leverage) for companies with a low effective tax rate (i.e., when HIGH_EFFECTIVE_TAX_RATE is equal to 0). Additionally, the coefficient of 
TABLE 4

Leverage and the Impact of Tax Reforms on Value:

Heterogeneous Effects by Country Legal Institutions

In Table 4, the dependent variable $d \mathrm{VNI}_{t} / A_{t-1}$ is the change in the market value of equity, minus any change in the book value of equity, all divided by lagged book assets. LOW TAX_EVASION refers to lower than median tax evasion among all sample countries. STRONG_ANTI_SELF_DEALING refers to greater than median anti-self-dealing index. All other variables are defined in Table 1. All regression models include country-year fixed effects. The regression models also include all the control variables and interactions in Table 2, although their coefficients are omitted for brevity. $t$-statistics based on standard errors adjusted for 2-way clustering (i.e., at the country-year and at the firm level) are shown in the parentheses below the coefficient estimates. ${ }^{*}{ }^{* *}$, and ${ }^{* * *}$ indicate significance at the $10 \%, 5 \%$, and $1 \%$ levels, respectively. All tax change variables, the GOOD_INSTITUTION indicator, and their interaction terms drop out of the models naturally due to the inclusion of country-year fixed effects.

\begin{tabular}{|c|c|c|c|c|}
\hline \multirow[b]{3}{*}{ Variable } & \multicolumn{4}{|c|}{ GOOD_INSTITUTION is: } \\
\hline & \multicolumn{2}{|c|}{ LOW_TAX_EVASION } & \multicolumn{2}{|c|}{$\begin{array}{c}\text { STRONG_ANTI_SELF_ } \\
\text { DEALING }\end{array}$} \\
\hline & 1 & 2 & 3 & 4 \\
\hline $\begin{array}{l}\text { LEVERAGE }_{t-1} \\
\times \text { CORPORATE_TAX_CHANGE } \\
\times \text { GOOD_INSTITUTION }\end{array}$ & $\begin{array}{l}11.174^{\star \star} \\
(2.28)\end{array}$ & $\begin{array}{l}20.252^{\star \star \star} \\
(3.11)\end{array}$ & $\begin{array}{l}10.473^{\star \star} \\
(2.04)\end{array}$ & $\begin{array}{l}18.161^{\star \star \star} \\
(2.74)\end{array}$ \\
\hline $\begin{array}{l}\text { LEVERAGE }_{t-1} \\
\quad \times \text { INTEREST_TAX_CHANGE } \\
\times \text { GOOD_INSTITUTION }\end{array}$ & & $\begin{array}{l}-16.572^{\star \star \star} \\
(-3.15)\end{array}$ & & $\begin{array}{l}-15.943^{* * *} \\
(-2.85)\end{array}$ \\
\hline $\begin{array}{l}\text { LEVERAGE }_{t-1} \\
\quad \times \text { DIVIDEND_TAX_CHANGE } \\
\times \text { GOOD_INSTITUTION }\end{array}$ & & $\begin{array}{l}4.379^{\star} \\
(1.72)\end{array}$ & & $\begin{array}{l}9.540^{* *} \\
(2.53)\end{array}$ \\
\hline $\begin{array}{l}\text { LEVERAGE }_{t-1} \\
\quad \times \text { CORPORATE_TAX_CHANGE }\end{array}$ & $\begin{array}{l}-1.331 \\
(-1.12)\end{array}$ & $\begin{array}{l}-2.889^{\star \star} \\
(-2.48)\end{array}$ & $\begin{array}{l}-1.017 \\
(-0.75)\end{array}$ & $\begin{array}{l}-1.586 \\
(-0.96)\end{array}$ \\
\hline $\begin{array}{l}\text { LEVERAGE }_{t-1} \\
\quad \times \text { INTEREST_TAX_CHANGE }\end{array}$ & & $\begin{array}{r}2.082 \\
(1.10)\end{array}$ & & $\begin{array}{r}1.037 \\
(0.51)\end{array}$ \\
\hline $\begin{array}{l}\text { LEVERAGE }_{t-1} \\
\quad \times \text { DIVIDEND_TAX_CHANGE }\end{array}$ & & $\begin{array}{l}0.568 \\
(0.64)\end{array}$ & & $\begin{array}{l}-4.449 \\
(-1.52)\end{array}$ \\
\hline $\begin{array}{l}E_{t} / A_{t-1} \\
\quad \times \text { CORPORATE_TAX_CHANGE } \\
\quad \times \text { GOOD_INSTITUTION }\end{array}$ & $\begin{array}{l}-35.290^{\star} \\
(-1.88)\end{array}$ & $\begin{array}{l}-36.147^{\star \star} \\
(-2.05)\end{array}$ & $\begin{array}{l}-29.285 \\
(-1.55)\end{array}$ & $\begin{array}{l}-30.170^{*} \\
(-1.71)\end{array}$ \\
\hline $\begin{array}{l}E_{t} / A_{t-1} \\
\quad \times \text { CORPORATE_TAX_CHANGE }\end{array}$ & $\begin{array}{l}-6.870 \\
(-0.99)\end{array}$ & $\begin{array}{l}-4.910 \\
(-0.64)\end{array}$ & $\begin{array}{l}-11.199 \\
(-1.52)\end{array}$ & $\begin{array}{l}-8.962 \\
(-1.13)\end{array}$ \\
\hline $\begin{array}{l}\text { LEVERAGE }_{t-1} \\
\quad \times \text { GOOD_INSTITUTION }^{-}\end{array}$ & $\begin{array}{l}0.322^{\star \star} \\
(2.28)\end{array}$ & $\begin{array}{l}0.344^{\star \star} \\
(2.34)\end{array}$ & $\begin{array}{l}0.353^{\star *} \\
(2.43)\end{array}$ & $\begin{array}{l}0.390^{\star * *} \\
(2.58)\end{array}$ \\
\hline$E_{t} / A_{t-1} \times$ GOOD_INSTITUTION & $\begin{array}{l}-0.985^{\star \star \star} \\
(-4.78)\end{array}$ & $\begin{array}{l}-0.951^{\text {** }} \\
(-4.99)\end{array}$ & $\begin{array}{l}-0.866^{\star * *} \\
(-3.67)\end{array}$ & 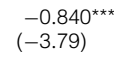 \\
\hline $\begin{array}{l}\text { Other controls (see Table 2) } \\
\text { Other interactions (see Table 2) } \\
\text { Country-year fixed effects }\end{array}$ & $\begin{array}{l}\text { Yes } \\
\text { Yes } \\
\text { Yes }\end{array}$ & $\begin{array}{l}\text { Yes } \\
\text { Yes } \\
\text { Yes }\end{array}$ & $\begin{array}{l}\text { Yes } \\
\text { Yes } \\
\text { Yes }\end{array}$ & $\begin{array}{l}\text { Yes } \\
\text { Yes } \\
\text { Yes }\end{array}$ \\
\hline $\begin{array}{l}\text { No. of obs. } \\
\text { Adj. } R^{2}\end{array}$ & $\begin{array}{r}203,676 \\
0.078 \\
\end{array}$ & $\begin{array}{r}203,241 \\
0.082\end{array}$ & $\begin{array}{r}203,676 \\
0.078\end{array}$ & $\begin{array}{r}203,241 \\
0.082\end{array}$ \\
\hline
\end{tabular}

the LEVERAGE $E_{t-1} \times$ CORPORATE_TAX_CHANGE $\times$ HIGH_EFFECTIVE_ TAX_RATE interaction reflects the incremental importance of a corporate tax change for companies with a HIGH_EFFECTIVE_TAX_RATE. The results indicate that, for companies with a HIGH_EFFECTIVE_TAX_RATE, reforms affect the value of debt tax shields more, compared with companies subject to a lower tax rate.

In regression 2 of Table 3, similar conclusions are reached when we define the effective tax rate as the sum of taxes paid during the 3-year period starting 2 years prior to the tax reform and ending the year of the reform, divided by the sum of pretax income in the same 3 years, following Dyreng, Hanlon, and Maydew (2008). In regression 3, we show that similar conclusions are reached when we use firm profitability (ROA) as a proxy for a firm's tax status. (We classify a firm 
as HIGH_ROA (equal to 1 ) if the firm's ROA is above its median value for the country and year, and 0 otherwise.)

In regression 4 of Table 3 , the coefficient on LEVERAGE $_{t-1} \times$ DIVIDEND_TAX_CHANGE reflects the importance of a DIVIDEND_TAX_CHANGE (i.e., the differential effect of DIVIDEND_TAX_CHANGES on value depending on financial leverage) for non-dividend payers (i.e., when DIVIDEND_PAYER $=0$ ). The results indicate that, for non-dividend payers, reforms that change the DIVIDEND_TAX rate do not significantly affect the value of a firm's equity. The

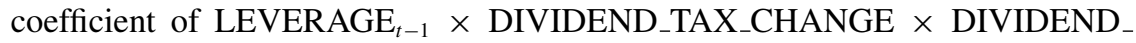
PAYER represents the incremental importance of a DIVIDEND_TAX_CHANGE for firms that pay dividends. The results indicate that DIVIDEND_TAX reforms have more impact on the value of payers (as compared with non-payers), although the difference between the two is not statistically significant at conventional levels $(t$-statistic $=1.52)$. The sum of the coefficients of LEVERAGE L $_{t-1}$ $\times$ DIVIDEND_TAX_CHANGE and LEVERAGE $t_{t-1} \times$ DIVIDEND_TAX $_{-}$ CHANGE $\times$ DIVIDEND_PAYER, or $3.57(t$-statistic $=1.91)$, reflects the importance of a DIVIDEND_TAX_CHANGE for dividend payers.

Taxes should have less impact on value in countries with high levels of tax evasion. In particular, if firms could evade taxes at no cost, taxes (and tax reforms) would have no impact on the equity value of firms; the same would apply to taxsheltering devices, such as debt. To investigate whether this is the case, we split countries into two groups based on the prevalence of tax evasion using the World Economic Forum's assessment of the prevalence of tax evasion in a country in 2002. This index is built from a survey of corporate executives' assessments of the prevalence of tax evasion in their countries. ${ }^{9,10}$ As predicted by a tax story, we find that tax reforms have little impact on equity values overall or through debt tax shields in countries with high levels of tax evasion (regressions 1 and 2 of Table 4). In particular, the LEVERAGE $\times$ CORPORATE_(PERSONAL)_TAX_CHANGE interactions, which reflect the impact of the reforms in countries with high tax evasion (i.e., when LOW_TAX_EVASION $=0$ ), generally lack statistical significance. In contrast, tax reforms have a significantly larger impact on equity values in countries with low levels of tax evasion, as documented by the statistically significant coefficients of the triple interaction terms LEVERAGE $\times$ CORPORATE_(PERSONAL)_TAX_CHANGE $\times$ LOW_TAX_EVASION.

Taxes (and debt tax shields) should also have less impact on equity values in countries where insiders shelter income from taxation through outright stealing (Desai, Dyck, and Zingales (2007)). To investigate whether this is the case, we employ Djankov, La Porta, and Lopez-de-Silanes's (2008) index of

\footnotetext{
${ }^{9}$ To investigate tax evasion within the United States, Guedhami and Pittman (2008) employ data compiled by the Transactional Records Access Clearinghouse. Those data include Internal Revenue Service sanctions against firms for tax evasion and tax fraud.

${ }^{10}$ Belgium, the Czech Republic, France, Germany, Greece, Hungary, Italy, Mexico, Poland, Portugal, Slovakia, South Korea, Spain, and Sweden have above-median levels of tax evasion, while Australia, Austria, Canada, Denmark, Finland, Iceland, Ireland, Japan, Luxembourg, the Netherlands, New Zealand, Norway, Switzerland, the United Kingdom, and the United States have median or below-median levels of tax evasion according to the measure developed by the World Economic Forum.
} 
anti-self-dealing, which measures the legal protection of minority shareholders against diversion of corporate wealth by insiders through self-dealing transactions. ${ }^{11}$ Less protection means that it is easier for insiders to steal, and this incentive to steal should be stronger when corporate tax rates are higher. Consistent with this story, we find that, when stealing is relatively easier, tax reforms and tax-sheltering devices appear to have no impact on equity values, as suggested by the lack of significance of the double interaction terms (i.e., LEVERAGE $\times$ CORPORATE_(PERSONAL)_TAX_CHANGE). ${ }^{12}$ However, on average, tax reforms and debt tax shields have a more significant impact on equity values in countries that highly restrict the ability of insiders to steal. Importantly, the results in this section mitigate the concern that our evidence may reflect something other than tax benefits, while providing support to a tax explanation of our findings.

\section{Event Study}

In the previous analyses, we included country-year FEs to control for changes in any omitted country-level factors that might affect all firms in a given country at a given point in time. A subtler concern is that the results might reflect omitted shocks that might occur in the year of a tax reform and that might affect various firms differently (specifically, through a leverage channel). To mitigate this concern, we employ an event study methodology. We focus on a narrow event window surrounding a tax reform news event. Selecting a narrow window enables us to filter out other value-relevant events unrelated to taxes.

Of course, this methodology has some limitations. In particular, we can focus only on tax reforms introduced as surprises. Further, we must be able to identify an event date. Finally, by focusing on a narrow window, we neglect the impact of any information related to the tax reform that is disseminated outside the event window. That is, we are able to only partially capture the impact of the tax reforms on value. With these caveats in mind, we undertake an event study of the price change observed around large corporate tax rate reductions. In particular, we focus on changes (reductions) in the top statutory corporate tax rate of at least 5 percentage points. ${ }^{13}$ Imposing these constraints allows us to focus on

\footnotetext{
${ }^{11}$ Australia, Belgium, Canada, Denmark, Finland, Ireland, Italy, Japan, New Zealand, Norway, Portugal, South Korea, the United Kingdom, and the United States have an above-median antiself-dealing index, while Austria, the Czech Republic, France, Germany, Greece, Hungary, Iceland, Luxembourg, Mexico, the Netherlands, Poland, Slovakia, Spain, Sweden, and Switzerland have a median or below-median anti-self-dealing index.

${ }^{12}$ Although the tax evasion index and the anti-self-dealing index are positively correlated $(\rho=0.62)$, there is still a fair amount of divergence between them. For example, nine countries involving 11,002 observations are classified as either high tax evasion and high anti-self-dealing countries or low tax evasion and low anti-self-dealing countries.

${ }^{13} \mathrm{We}$ focus on relatively large reforms in order to isolate events that should have a meaningful effect on equity values. (Presumably, very minor tax reforms are less likely to have detectable impacts on value.) We focus on tax reductions because those represent the vast majority of tax reforms. We focus only on reforms affecting corporate tax rates for two reasons. First, corporate tax rates (as opposed to personal tax rates) have historically formed the basis of pedagogy in finance. Second, identifying the exact news disclosure dates of the tax reforms through news searches is a painfully lengthy undertaking. For example, a preliminary unrestricted search of all tax reforms in Factiva using the keywords "(tax w/5 reform) or (tax rate w/5 change)" during the period 1981-2009 returns 266,304 news articles. Focusing on one specific type of reform makes the identification process less challenging.
} 
an initial sample of 28 country-years in which a large corporate tax rate reduction occurred. ${ }^{14}$

For each of those 28 large corporate tax reductions, we conduct keyword searches in Factiva to identify the date of i) the first rumor about the tax rate reduction in the press, ii) a major "official" statement made by government officials about a specific proposal of a reduction in the corporate tax rate, and iii) the final approval of the law introducing a tax rate reduction. These keyword searches yield a sample of 58 announcements related to 23 specific tax reforms. The date and a brief description of each announcement are reported in Panel A of Table 5.

For each firm in those countries with available stock price data, we compute a 5-day CUMULATIVE_STOCK_RETURN (in US\$ terms) over the interval beginning 2 days prior to and ending 2 days after the tax change news, $\sum_{t=-2}^{+2} R_{i, t}$. To be consistent with the analysis in the previous tables, we continue to scale the independent variables by the book value of total assets. Because of this scaling, the dependent variable also needs to be scaled by the book value of assets. ${ }^{15}$

As shown in Panel B of Table 5, firms' equity value increases, on average, around news of tax reductions. For the average firm, we document a $0.46 \%$ increase in value. As expected, among different types of events, the first rumor of a tax cut (RUMOR) is associated with the largest increase in value (1.40\%).

Given our focus on large changes in tax rates, these numbers may appear small. There are several reasons for this. First, although we focus on the release of new information related to a specific tax change, that information typically relates to partial rather than full events. Second, the typical firm is levered so that its income is, at least in part, shielded from taxation. With those caveats in mind, we, nevertheless, find that corporate tax changes do affect equity prices. In the lower part of Panel B of Table 5, we further document that a tax cut has a larger impact on the equity value of firms with low leverage and that its impact declines as leverage increases.

Table 6 presents some regression results using the 5-day cumulative stock return computed in Table 5 as a dependent variable. Regression 1 confirms a positive sign for the interaction between leverage and corporate tax reforms, after controlling for a number of firm-level attributes. Regression 2 repeats the specification in regression 1 using only the first "news event" for each reform, which should be the most important news release for each reform. The results are consistent with those in regression 1. Regression 3 shows that the results are robust to controlling for possible reform-specific omitted variables through the inclusion of reform FEs. In regression 4, we exclude corporate tax reforms involving a simultaneous large (i.e., larger than 5 percentage points) change in personal tax rates on interest or dividend income. While the resulting sample size is substantially smaller than in the previous columns, our results are qualitatively unchanged.

\footnotetext{
${ }^{14}$ By comparison, there are only four large corporate tax rate increases in the sample.

${ }^{15}$ Qualitatively similar results obtain if we scale the variables by the lagged market value of equity.
} 
TABLE 5

\section{Summary Statistics of Corporate Tax Reduction Event Tests}

Panel A of Table 5 lists the dates, types, and other information about the news announcements on major corporate tax reductions in OECD countries during 1980-2009. Major corporate tax reductions are tax reductions of $5 \%$ or more. News type RUMOR indicates news about a possible upcoming tax reform without much detail. News type INTENTION indicates news about a government's or legislator's stated intention to initiate a tax reform with fair amount of detail. News type LAW indicates news about the passage of the tax law. Panel B provides summary statistics of the 5-day cumulative stock returns for all firms, by news type and by book leverage. The 5-day CUMULATIVE_STOCK_RETURN is the sum of daily stock returns in the 5 -day window surrounding a tax reduction event (between day -2 and day 2), multiplied by the firm's market value of equity and divided by the book value of total assets. LEVERAGE $t-1$ is total interest-bearing debt divided by total assets at the beginning of the reform year. ${ }^{*},{ }^{* \star}$, and ${ }^{* \star *}$ indicate significance at the $10 \%, 5 \%$, and $1 \%$ levels, respectively.

Panel A. Description of News Events around Corporate Tax Changes

\begin{tabular}{|c|c|c|c|c|c|c|}
\hline $\begin{array}{l}\text { News } \\
\text { Counts }\end{array}$ & Country & $\begin{array}{l}\text { Year Tax Change } \\
\text { Implemented }\end{array}$ & News Type & News Date & $\begin{array}{c}\text { Old } \\
\text { Tax Rate }\end{array}$ & $\begin{array}{c}\text { New } \\
\text { Tax Rate }\end{array}$ \\
\hline 1 & Australia & 1988 & RUMOR & 9/17/1987 & 49.00 & 39.00 \\
\hline 2 & Australia & 1988 & INTENTION & $2 / 14 / 1988$ & 49.00 & 39.00 \\
\hline 3 & Australia & 1988 & LAW & 5/26/1988 & 49.00 & 39.00 \\
\hline 4 & Australia & 1988 & LAW & $5 / 29 / 1988$ & 49.00 & 39.00 \\
\hline 5 & Australia & 1993 & RUMOR & 2/10/1993 & 39.00 & 33.00 \\
\hline 6 & Austria & 1989 & LAW & $3 / 7 / 1988$ & 55.00 & 30.00 \\
\hline 7 & Austria & 2005 & RUMOR & $3 / 7 / 2003$ & 34.00 & 25.00 \\
\hline 8 & Austria & 2005 & RUMOR & $1 / 9 / 2004$ & 34.00 & 25.00 \\
\hline 9 & Austria & 2005 & LAW & 3/23/2004 & 34.00 & 25.00 \\
\hline 10 & Belgium & 2003 & RUMOR & $10 / 4 / 2001$ & 40.17 & 33.99 \\
\hline 11 & Belgium & 2003 & INTENTION & 10/9/2001 & 40.17 & 33.99 \\
\hline 12 & Belgium & 2003 & INTENTION & 3/26/2002 & 40.17 & 33.99 \\
\hline 13 & Belgium & 2003 & LAW & 12/11/2002 & 40.17 & 33.99 \\
\hline 14 & Denmark & 1990 & INTENTION & 5/12/1989 & 50.00 & 40.00 \\
\hline 15 & Denmark & 1990 & LAW & $12 / 1 / 1989$ & 50.00 & 40.00 \\
\hline 16 & Finland & 1993 & INTENTION & 9/25/1992 & 39.00 & 25.00 \\
\hline 17 & France & 1992 & INTENTION & $8 / 29 / 1990$ & 42.00 & 34.00 \\
\hline 18 & France & 1992 & LAW & 6/1/1991 & 42.00 & 34.00 \\
\hline 19 & Germany & 1990 & INTENTION & $6 / 22 / 1989$ & 60.00 & 54.55 \\
\hline 20 & Germany & 1990 & INTENTION & 8/28/1990 & 60.00 & 54.55 \\
\hline 21 & Germany & 2001 & INTENTION & 12/7/1999 & 52.03 & 38.90 \\
\hline 22 & Germany & 2001 & INTENTION & 12/21/1999 & 52.03 & 38.90 \\
\hline 23 & Germany & 2001 & INTENTION & 2/9/2000 & 52.03 & 38.90 \\
\hline 24 & Germany & 2001 & LAW & $7 / 14 / 2000$ & 52.03 & 38.90 \\
\hline 25 & Germany & 2008 & INTENTION & $3 / 14 / 2007$ & 38.90 & 30.18 \\
\hline 26 & Germany & 2008 & LAW & $7 / 6 / 2007$ & 38.90 & 30.18 \\
\hline 27 & Hungary & 1995 & RUMOR & $10 / 26 / 1994$ & 36.00 & 18.00 \\
\hline 28 & Hungary & 1995 & INTENTION & 10/28/1994 & 36.00 & 18.00 \\
\hline 29 & Italy & 1998 & INTENTION & 5/3/1996 & 53.20 & 41.25 \\
\hline 30 & Italy & 1998 & INTENTION & 9/13/1997 & 53.20 & 41.25 \\
\hline 31 & Italy & 1998 & INTENTION & $10 / 15 / 1997$ & 53.20 & 41.25 \\
\hline 32 & Italy & 1998 & LAW & $12 / 9 / 1997$ & 53.20 & 41.25 \\
\hline 33 & Italy & 2008 & RUMOR & $8 / 28 / 2007$ & 37.25 & 31.40 \\
\hline 34 & Italy & 2008 & INTENTION & $9 / 25 / 2007$ & 37.25 & 31.40 \\
\hline 35 & Italy & 2008 & LAW & $9 / 28 / 2007$ & 37.25 & 31.40 \\
\hline 36 & Italy & 2008 & LAW & $12 / 5 / 2007$ & 37.25 & 31.40 \\
\hline 37 & Japan & 2000 & INTENTION & 5/17/1998 & 48.00 & 42.00 \\
\hline 38 & New Zealand & 1988 & INTENTION & 11/19/1987 & 48.00 & 28.00 \\
\hline 39 & New Zealand & 1988 & INTENTION & $12 / 17 / 1987$ & 48.00 & 28.00 \\
\hline 40 & New Zealand & 1988 & LAW & 2/10/1988 & 48.00 & 28.00 \\
\hline 41 & Norway & 1992 & INTENTION & $5 / 21 / 1990$ & 50.80 & 28.00 \\
\hline 42 & Norway & 1992 & INTENTION & $4 / 10 / 1991$ & 50.80 & 28.00 \\
\hline 43 & Poland & 2004 & RUMOR & $2 / 24 / 2003$ & 27.00 & 19.00 \\
\hline 44 & Poland & 2004 & INTENTION & 4/10/2003 & 27.00 & 19.00 \\
\hline 45 & Poland & 2004 & INTENTION & 4/24/2003 & 27.00 & 19.00 \\
\hline 46 & Poland & 2004 & LAW & 6/17/2003 & 27.00 & 19.00 \\
\hline 47 & Portugal & 2004 & INTENTION & 3/17/2002 & 33.00 & 27.50 \\
\hline 48 & Portugal & 2004 & INTENTION & $12 / 19 / 2002$ & 33.00 & 27.50 \\
\hline 49 & Portugal & 2004 & INTENTION & 11/1/2003 & 33.00 & 27.50 \\
\hline 50 & Portugal & 2004 & LAW & $11 / 21 / 2003$ & 33.00 & 27.50 \\
\hline 51 & Slovakia & 2000 & INTENTION & $5 / 19 / 1998$ & 40.00 & 29.00 \\
\hline 52 & Slovakia & 2000 & INTENTION & $11 / 1 / 1999$ & 40.00 & 29.00 \\
\hline 53 & Slovakia & 2000 & LAW & 11/24/1999 & 40.00 & 29.00 \\
\hline 54 & Slovakia & 2004 & INTENTION & 3/26/2003 & 25.00 & 19.00 \\
\hline 55 & Sweden & 1990 & INTENTION & 10/3/1989 & 60.10 & 53.00 \\
\hline 56 & United States & 1987 & INTENTION & $11 / 28 / 1984$ & 49.82 & 44.18 \\
\hline 57 & United States & 1987 & INTENTION & $5 / 30 / 1985$ & 49.82 & 44.18 \\
\hline 58 & United States & 1987 & LAW & $6 / 24 / 1986$ & 49.82 & 44.18 \\
\hline
\end{tabular}

,


TABLE 5 (continued)

Summary Statistics of Corporate Tax Reduction Event Tests

Panel B. Five-Day CUMULATIVE_STOCK_RETURN (in \%), Total and by Leverage

\begin{tabular}{|c|c|c|}
\hline & No. of Obs. & Mean \\
\hline All firms & 13,194 & $0.46^{\star \star \star}$ \\
\hline $\begin{array}{l}\text { By news type } \\
\text { RUMOR } \\
\text { INTENTION } \\
\text { LAW }\end{array}$ & $\begin{array}{r}725 \\
8,705 \\
3,764\end{array}$ & $\begin{array}{l}1.40^{\star \star \star x} \\
0.25^{\star \star \star x} \\
0.78^{\star \star \star *}\end{array}$ \\
\hline $\begin{array}{c}\text { By LEVERAGE } t-1 \\
\text { Below median } \\
\text { Above median } \\
\text { Above-Below }\end{array}$ & $\begin{array}{l}6,597 \\
6,597\end{array}$ & $\begin{array}{c}0.79^{\star \star \star \star} \\
0.14^{\star \star \star} \\
-0.65^{\star \star \star}\end{array}$ \\
\hline
\end{tabular}

\section{Propensity Score Matching}

We next assess the robustness of our results to the use of a propensity score matching methodology (Rosenbaum and Rubin (1983)). As in the previous section, we continue to focus on large tax-reducing reforms. For each large corporate tax reform, we use the propensity score matching procedure to match firms with above-median leverage with firms with below-median leverage, in the same country and year, and with characteristics that are otherwise similar to those of highly levered firms. The propensity score is estimated as a function of all the firm-level control variables in Table 2. We then compare the two groups with respect to change in the value of equity relative to the year-end prior to the tax reform. As the set of control firms is designed to be nearly identical to the treatment group in terms of observables (with the exception of leverage), the average change in the value of equity should be similar between the two groups if debt tax shields did not affect the value of equity. To ensure that any differences between the two sets of firms are small, we require that the difference between the propensity scores does not exceed $1 \%$ in absolute value. ${ }^{16}$

The results are reported in Table 7 . There, we compare the change in value for firms with above-median leverage to that of control firms with below-median leverage. Both sets of firms are taken from the same country and year. The results indicate that the change in value during the year of the reform is significantly greater for firms with low leverage. Highly levered firms are, instead, only marginally affected by the tax reform. The difference in the change in value (scaled by total assets) between the two sets of firms is equal to $-4.55 \%$, which is statistically significant with a $p$-value of 0.03 . These results are in line with our earlier evidence. We conclude that our earlier results do not appear to be driven by the specific econometric methodology used.

\section{Economic Significance}

Figure 1 illustrates the approach we use to assess the economic significance of the results. It displays the marginal benefit (MB) and the marginal cost (MC) curves for two firms based in a country undergoing a corporate tax reform. At the

\footnotetext{
${ }^{16}$ We find $\log$ sales to differ statistically between the treatment and the control groups of firms. However, we note that the difference is rather small in economic magnitude.
} 
TABLE 6

Leverage, Tax Changes, and 5-Day Cumulative Stock Returns

The dependent variable in Table 6 is the 5-day cumulative stock return (in \%) around news events on major corporate tax reforms, as defined in Table 5. Major corporate tax reforms are corporate tax reductions of 5\% or more. TAX_CHANGE is the new tax rate of a reform minus the old tax rate. All variables are defined in Table 1, and the independent variables are measured in the year (or year-end) prior to the event dates. $t$-statistics based on standard errors adjusted for 2-way clustering (i.e., at the country-year and at the firm level) are shown in the parentheses below the coefficient estimates. *, ${ }^{* *}$, and ${ }^{* \star *}$ indicate significance at the $10 \%, 5 \%$, and $1 \%$ levels, respectively.

\begin{tabular}{|c|c|c|c|c|}
\hline & Full Sample & $\begin{array}{l}\text { Subsample of } \\
\text { First "News } \\
\text { Events" }\end{array}$ & $\begin{array}{l}\text { Full Sample with } \\
\text { Reform FEs }\end{array}$ & $\begin{array}{l}\text { Subsample of "Pure" } \\
\text { Corporate Tax } \\
\text { Reforms with } \\
\text { Reform FEs } \\
4\end{array}$ \\
\hline Variable & 1 & 2 & 3 & \\
\hline LEVERAGE $_{t-1} \times$ TAX_CHANGE & $\begin{array}{l}20.446^{*} \\
(1.65)\end{array}$ & $\begin{array}{l}27.575^{\star} \\
(1.77)\end{array}$ & $\begin{array}{l}22.272^{\star * \star} \\
(2.85)\end{array}$ & $\begin{array}{l}10.643^{\star *} \\
(2.11)\end{array}$ \\
\hline$E_{t} / A_{t-1} \times$ TAX_CHANGE & $\begin{array}{l}-99.272 \\
(-1.29)\end{array}$ & $\begin{array}{c}-149.439 \\
(-1.51)\end{array}$ & $\begin{array}{l}-81.119 \\
(-1.16)\end{array}$ & $\begin{array}{l}-46.910^{* \star *} \\
(-3.49)\end{array}$ \\
\hline$d \mathrm{DEBT}_{t} / \mathrm{A}_{t-1} \times$ TAX_CHANGE & $\begin{array}{l}-14.099 \\
(-0.41)\end{array}$ & $\begin{array}{c}33.774 \\
(1.57)\end{array}$ & $\begin{array}{l}-21.512 \\
(-0.61)\end{array}$ & $\begin{array}{l}12.431^{*} \\
(1.92)\end{array}$ \\
\hline$d \mathrm{DEBT}_{t} / \mathrm{A}_{t-1}$ & $\begin{array}{l}-0.372 \\
(-0.14)\end{array}$ & $\begin{array}{r}3.121 \\
(1.45)\end{array}$ & $\begin{array}{l}-1.518 \\
(-0.61)\end{array}$ & $\begin{array}{l}9.202^{\star \star *} \\
(6.15)\end{array}$ \\
\hline $\ln \left(\right.$ SALES $\left._{t}\right)$ & $\begin{array}{l}0.176^{\star \star \star} \\
(2.77)\end{array}$ & $\begin{array}{l}0.159^{\star} \\
(1.87)\end{array}$ & $\begin{array}{l}0.188^{* \star \star} \\
(3.12)\end{array}$ & $\begin{array}{l}0.065 \\
(0.80)\end{array}$ \\
\hline$\left(\mathrm{M} \_\mathrm{B}\right)_{t-1}$ & $\begin{array}{r}0.651 \\
(1.24)\end{array}$ & $\begin{array}{l}0.223 \\
(0.30)\end{array}$ & $\begin{array}{c}0.623 \\
(1.16)\end{array}$ & $\begin{array}{l}-0.012 \\
(-0.05)\end{array}$ \\
\hline$d E_{t} / A_{t-1}$ & $\begin{array}{l}-0.541 \\
(-0.33)\end{array}$ & $\begin{array}{l}-0.253 \\
(-0.09)\end{array}$ & $\begin{array}{l}-0.370 \\
(-0.23)\end{array}$ & $\begin{array}{l}2.169^{\star \star *} \\
(5.57)\end{array}$ \\
\hline$d \mathrm{NPPE}_{t} / A_{t-1}$ & $\begin{array}{r}1.141 \\
(1.46)\end{array}$ & $\begin{array}{r}0.344 \\
(0.24)\end{array}$ & $\begin{array}{c}0.226 \\
(0.34)\end{array}$ & $\begin{array}{l}-0.292 \\
(-1.62)\end{array}$ \\
\hline$d \mathrm{RD}_{t} / A_{t-1}$ & $\begin{array}{l}-28.976^{*} \\
(-1.90)\end{array}$ & $\begin{array}{l}-23.750 \\
(-1.10)\end{array}$ & $\begin{array}{l}-26.111^{*} \\
(-1.79)\end{array}$ & $\begin{array}{r}1.610 \\
(0.43)\end{array}$ \\
\hline LEVERAGE $_{t-1}$ & $\begin{array}{r}1.194 \\
(1.11)\end{array}$ & $\begin{array}{r}1.190 \\
(1.07)\end{array}$ & $\begin{array}{l}1.786^{\star \star} \\
(2.41)\end{array}$ & $\begin{array}{l}1.376 \\
(0.37)\end{array}$ \\
\hline$E_{t} / A_{t-1}$ & $\begin{array}{l}-3.855 \\
(-0.51)\end{array}$ & $\begin{array}{l}-9.484 \\
(-0.93)\end{array}$ & $\begin{array}{l}-1.992 \\
(-0.30)\end{array}$ & $\begin{array}{l}13.289^{* * *} \\
(3.07)\end{array}$ \\
\hline CYCLICAL_INDUSTRY & $\begin{array}{l}-0.470^{*} \\
(-1.65)\end{array}$ & $\begin{array}{l}-0.425^{\star} \\
(-1.90)\end{array}$ & $\begin{array}{l}-0.355 \\
(-1.36)\end{array}$ & $\begin{array}{l}-0.054 \\
(-0.24)\end{array}$ \\
\hline TAX_CHANGE & $\begin{array}{l}-8.955 \\
(-1.05)\end{array}$ & $\begin{array}{l}-16.253^{*} \\
(-1.73)\end{array}$ & & \\
\hline Fixed effects & None & None & $\begin{array}{c}\text { Tax Reform } \\
\text { Event }\end{array}$ & $\begin{array}{c}\text { Tax Reform } \\
\text { Event }\end{array}$ \\
\hline $\begin{array}{l}\text { No. of obs. } \\
\text { Adj. } R^{2}\end{array}$ & $\begin{array}{r}11,252 \\
0.034\end{array}$ & $\begin{array}{l}5,421 \\
0.024\end{array}$ & $\begin{array}{r}11,252 \\
0.082\end{array}$ & $\begin{array}{l}3,203 \\
0.029\end{array}$ \\
\hline
\end{tabular}

optimal initial leverage level of $(D / A)_{0}^{H}$, the gross tax benefit of debt for firm $H$ is represented by the rectangle $O(D / A)_{0}^{H} C D$, and the net (of debt costs) tax benefit of debt equals the area $O C D$, where $O$ represents the origin of both axes. The corporate tax rate is reduced from $T_{C_{0}}$ to $T_{C_{1}}$. As a consequence, the MB curve moves down and the optimal amount of debt changes to the point where the marginal cost is equal to the new marginal benefit. Considering the change in the cost of debt due to leverage adjustment predicted to occur in response to the reform, the area $A N C D(A M F D)$ represents the change in the net (of debt costs) tax benefits of debt financing for firm $H(L)$.

Assuming the marginal cost function to be linear in the band between $T_{C_{0}}$ and $T_{C_{1}}$ without loss of generality, the change in the net (of debt costs) tax benefits of debt financing should theoretically be equal to $\left[D_{0}^{H} \times\left(\Delta T_{C}\right)+\right.$ $\left.\left(\Delta D^{H}\right) \times\left(\Delta T_{C}\right) / 2\right] / A$ for firm $H$ and $\left[D_{0}^{L} \times\left(\Delta T_{C}\right)+\left(\Delta D^{L}\right) \times\left(\Delta T_{C}\right) / 2\right] / A$ for firm $L$. 


\section{TABLE 7}

Leverage and the Impact of Tax Reforms on Value:

Propensity Score Matching Results

\begin{tabular}{|c|c|c|c|c|}
\hline Variable & $\begin{array}{l}\text { High Leverage } \\
(N=2,483) \\
\end{array}$ & $\begin{array}{l}\text { Low Leverage } \\
(N=2,483)\end{array}$ & $\begin{array}{c}\text { Diff. } \\
\text { (High - Low } \\
\text { Leverage) } \\
\end{array}$ & $\underline{p \text {-Value }}$ \\
\hline $\begin{array}{l}\text { Matching variables } \\
\text { In }\left(\mathrm{SALES}_{t}\right) \\
\left(\mathrm{M} \mathrm{B}_{t-1}\right. \\
d E_{t} / A_{t-1} \\
d \mathrm{NPPE}_{t} / A_{t-1} \\
d \mathrm{RD}_{t} / A_{t-1} \\
E_{t} / A_{t-1}\end{array}$ & $\begin{array}{r}12.6836 \\
1.3723 \\
0.0072 \\
0.0001 \\
0.0019 \\
0.0763\end{array}$ & $\begin{array}{r}12.7930 \\
1.4059 \\
0.0061 \\
0.0014 \\
0.0019 \\
0.0751\end{array}$ & $\begin{array}{r}-0.1094 \\
-0.0337 \\
0.0010 \\
-0.0013 \\
0.0000 \\
0.0011\end{array}$ & $\begin{array}{l}0.02 \\
0.29 \\
0.74 \\
0.38 \\
0.91 \\
0.72\end{array}$ \\
\hline $\begin{array}{l}\text { CYCLICAL_INDUSTRY } \\
d \mathrm{DEBT}_{t} / A_{t-1}\end{array}$ & $\begin{array}{l}0.7825 \\
0.0256\end{array}$ & $\begin{array}{l}0.7757 \\
0.0290\end{array}$ & $\begin{array}{r}0.0068 \\
-0.0034\end{array}$ & $\begin{array}{l}0.56 \\
0.44\end{array}$ \\
\hline $\begin{array}{l}\text { Other variables } \\
\text { LEVERAGE }_{t-1}\end{array}$ & 0.3693 & 0.1029 & 0.2664 & 0.00 \\
\hline $\begin{array}{l}\text { Value change } \\
d \mathrm{VNI}_{t} / A_{t-1} \\
(p \text {-value })\end{array}$ & $\begin{array}{c}-0.0199 \\
(0.11)\end{array}$ & $\begin{array}{l}0.0256 \\
(0.13)\end{array}$ & $-0.0455^{\star \star}$ & 0.03 \\
\hline
\end{tabular}

We compare the highly levered firm, $H$, to a second firm with low leverage, $L$. Both firms undergo the same tax reform. Focusing on the difference in the change in value experienced by two firms allows us to remove the impact of contaminating events that equally affect the value of different firms. The difference in the theoretical value of the (net) tax benefits of debt financing between firm $H$ and firm $L$ corresponds to the area $M N C F$ in Figure 1. To assess the economic significance of the results, in the rest of this section, we compare the empirical estimate of this area with its theoretical benchmark.

To empirically estimate the market value of debt tax shields, we rely on both the propensity score matching and the event study results. A major benefit of the propensity score results, which are based on an extended (1-year) event window, is that they are more likely to fully capture the impact of the tax reforms on value. However, if the event window was contaminated by other events that affect value through a leverage channel, the propensity score results may overstate the economic impact of tax reforms. The empirical implementation of our test relies on two presumptions: i) That the two firms optimize their capital structure and ii) that there are no unobserved differences beyond those captured by the matching that would also predict a different response to the tax reform. If these conditions are not met, the interpretation of the economic significance results is potentially less unambiguous.

With this caveat in mind, we note that the tax reforms covered in Table 7 result in an average reduction in the corporate tax rate, $\delta T_{C}$, of 6.71 percentage points. During the 1-year interval surrounding the tax reform, the difference in the change in value (scaled by total assets) between high- and low-leverage firms 
FIGURE 1

Marginal Benefit and Marginal Cost of a Given Amount of Debt, $D$

Figure 1 shows the marginal benefit (MB) (and the marginal cost $(\mathrm{MC})$ ) curves for a firm in a country undergoing a corporate tax reform. The corporate tax rate is reduced from $T_{C_{0}}$ to $T_{C_{1}}$.

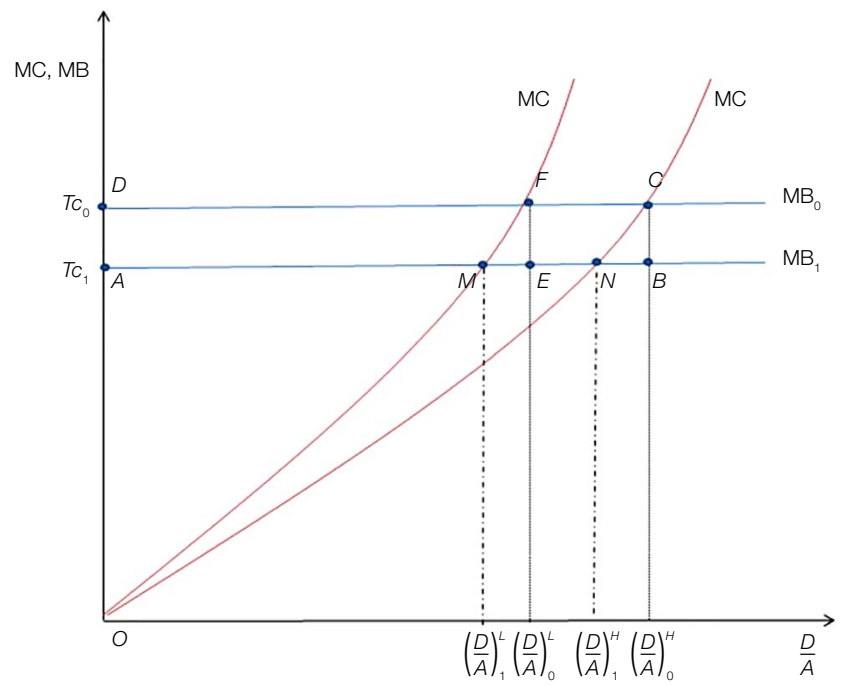

is equal to $-4.55 \%$ (with a $p$-value of 0.03 ). Its theoretical benchmark is equal to $-1.78 \%$. Although our empirical estimate is larger in magnitude, we find it to be statistically indistinguishable from its theoretical benchmark ( $p$-value of the difference $=0.19$ ).

We next turn to the event study results. The event study provides a lower bound for the economic significance of the results. This occurs because, despite our best efforts, our collected news announcements may not capture all means through which information about a tax reform is disseminated. At the same time, a major benefit of the event study is that using narrow event windows enables us to exclude a number of (unspecified) contaminating events that might broadly overlap with tax reforms and potentially affect value through a leverage channel.

With this in mind, we employ the results in Panel B of Table 5 to assess the economic magnitude of the tax benefits of debt. The tax reforms covered in the event study result in an average reduction in the corporate tax rate, $\delta T_{C}$, of 8.06 percentage points. The difference in the change in value (scaled by total assets) between high- and low-leverage firms is equal to $-0.65 \%$ (i.e., $0.14 \%-$ $0.79 \%$ ) during the 5-day interval surrounding these tax reforms. Recalling that, on average, we were able to identify 2.52 news releases for each reform (i.e., $58 / 23$ ), the aggregate change in value corresponding to each reform is roughly $-1.64 \%$ (i.e., $-0.65 \% \times 58 / 23$ ). By comparison, the theoretical benchmark for the difference in the change in value (scaled by total assets) between the two sets of firms is $-2.33 \%$. 
While the event study results yield an estimate that is lower than the theoretical benchmark, this result is expected as we are able to only partially capture the value of the tax reforms. Importantly, our propensity score matching results, which are more likely to capture the full impact of the tax reforms, yield an empirical estimate of the tax benefits of debt that is statistically indistinguishable from the theoretical benchmark. Based on these results, it appears that the tax savings associated with the deductibility of interest payments are in line with the theoretical predictions under a traditional approach. An extension of these results implies that, for the average firm in the sample with a leverage ratio of $23.92 \%$ and subject to the average corporate tax rate of $38.41 \%$, the gross tax benefits of debt are equal to $D / A \times T_{C}$. This corresponds to $9.19 \%$ (i.e., $23.92 \% \times 38.41 \%$ ) of the book value of assets.

\section{Robustness Tests}

In this section, we investigate the robustness of our results.

\section{A. Differences in the All-Equity Cost of Capital across Firms}

In our tests, we rely on the presumption that the all-equity cost of capital is the same across all firms. We do so because the estimation of the cost of capital presents substantial empirical challenges. In this section, we allow the cost of capital to vary across firms in different industries. We do so by reestimating equation (2) in Table 2 separately for each Fama-French industry. ${ }^{17}$ Table 8 summarizes the results using a Fama-MacBeth (1973) approach. More specifically, within each industry, we first run the regression model of value change on the interaction between lagged leverage and tax changes, as well as control variables. We then compute the average of the coefficients on the

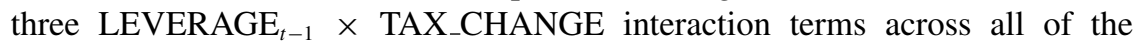
industry-level specifications from the first step and test their statistical significance based on standard errors computed from these industry-level coefficients. As the

\section{TABLE 8}

Leverage and the Impact of Tax Reforms on Value: Industry-by-Industry Regressions

For each of the Fama-French 30 industries (except tobacco, utilities, financial, and "everything else"), we run a regression of $d \mathrm{VNI}_{t} / A_{t-1}$, the annual change in the market value of equity, minus any change in the book value of equity (scaled by lagged book assets), on the interactions between leverage and corporate and personal tax changes, firmlevel control variables, and country-year FEs. Table 8 reports the average values and the test statistics of the regression coefficients on the three main tax interaction variables: LEVERAGE $t_{t-1} \times$ CORPORATE_TAX_CHANGE, LEVERAGE $_{t-1} \times$

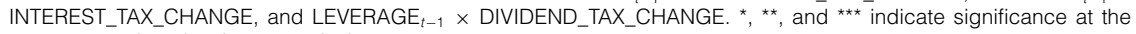
$10 \%, 5 \%$, and $1 \%$ levels, respectively.

\begin{tabular}{lc} 
Coefficient of: & $\begin{array}{c}\text { LEVERAGE }_{t-1} \\
\text { CORPORATE_TAX_ } \\
\text { Mean }\end{array}$ \\
\cline { 2 - 2 }$t$-statistic & $\begin{array}{c}7.883^{\star \star \star} \\
(2.71)\end{array}$ \\
\hline
\end{tabular}

\begin{tabular}{c} 
LEVERAGE $_{t-1}$ \\
$\times$ INTEREST_TAX_- \\
\hline CHANGE \\
\hline $\begin{array}{l}-8.461^{* *} \\
(-2.45)\end{array}$
\end{tabular}

LEVERAGE $_{t-1}$ $\times$ DIVIDEND_TAX_ CHANGE

$3.946^{\star *}$ (2.27)

\footnotetext{
17،"Tobacco" and the "everything else" industry drop out due to lack of observations.
} 
results in Table 8 show, our earlier conclusions are unchanged if we allow the all-equity cost of capital to vary across industries. Importantly, our earlier conclusions do not appear to be driven by assuming the same discount rate across firms.

\section{B. Results by Reform}

Because the data set contains a number of corporate tax cuts, we can estimate not only the mean treatment effect but also the impact of each individual reform on the value of equity. To do so, we run separate firm-level regressions of the change in the equity value for each large reform resulting in a reduction in the corporate tax rate of 5 percentage points or more. (Those regressions cannot include interaction terms due to perfect multicollinearity.) We run these regressions for 25 large reforms involving countries in which we have at least 12 firms. The results are reported in Table 9. In these regressions, the coefficient of interest is that of LEVERAGE ${ }_{t-1}$. Given that we are focusing on tax cuts, this coefficient is expected to be negative. Admittedly, analyzing reforms individually has its own limitations. For example, the coefficient estimate of LEVERAGE $_{t-1}$ will be

TABLE 9

Reform-by-Reform Regressions

For each large corporate tax-reducing reform, we run a regression of $d \mathrm{VNI}_{t} / A_{t-1}$, the annual change in the market value of equity, minus any change in the book value of equity (scaled by lagged book assets), on lagged leverage and control variables. Panel A of Table 9 reports the average values and the test statistics of the regression coefficients on lagged leverage, while Panel B reports these coefficients by reform. A large corporate tax-reducing reform is a reform resulting in a reduction in the corporate tax rate of 5 percentage points or more. *, **, and ${ }^{* \star *}$ indicate significance at the $10 \%, 5 \%$, and $1 \%$ levels, respectively.

Panel A. Summary of the Coefficients of LEVERAGE $E_{t-1}$

$\begin{array}{lc}\text { Mean coefficient of LEVERAGE } & -0.20^{* *} \\ t \text {-statistic } & (-2.20) \\ \text { Number of reforms with coefficients }>0 & 7 \\ \text { Number of reforms with coefficients }<0 & 18\end{array}$

Panel B. Coefficients on LEVERAGE $E_{t-1}$ for Each Reform

Corporate Tax-Reducing Reform In:

\begin{tabular}{|c|c|c|}
\hline Country & Year & No. of Obs. \\
\hline Australia & 1988 & 64 \\
\hline Australia & 1993 & 150 \\
\hline Austria & 1989 & 31 \\
\hline Austria & 2005 & 53 \\
\hline Belgium & 2003 & 79 \\
\hline Denmark & 1990 & 79 \\
\hline Finland & 1993 & 67 \\
\hline France & 1986 & 131 \\
\hline France & 1992 & 378 \\
\hline Germany & 1990 & 247 \\
\hline Germany & 2001 & 422 \\
\hline Germany & 2008 & 555 \\
\hline Italy & 1998 & 101 \\
\hline Italy & 2008 & 195 \\
\hline Japan & 2000 & 2,217 \\
\hline New Zealand & 1988 & 12 \\
\hline Norway & 1992 & 66 \\
\hline Poland & 2004 & 75 \\
\hline Portugal & 2004 & 48 \\
\hline Sweden & 1990 & 68 \\
\hline Sweden & 1991 & 73 \\
\hline United Kingdom & 1984 & 251 \\
\hline United Kingdom & 1986 & 331 \\
\hline United States & 1987 & 1,326 \\
\hline United States & 1988 & 1,340 \\
\hline
\end{tabular}

\begin{tabular}{cc}
$\begin{array}{c}\text { Coefficient of } \\
\text { LEVERAGE }_{t-1}\end{array}$ & $\begin{array}{c}t \text {-Statistic of } \\
\text { LEVERAGE }_{t-1}\end{array}$ \\
\cline { 2 - 2 }$-0.73^{\star \star}$ & $(-2.25)$ \\
$-0.80^{\star \star}$ & $(-2.46)$ \\
-0.62 & $(-0.86)$ \\
-1.47 & $(-0.73)$ \\
-0.11 & $(-0.55)$ \\
-0.19 & $(-0.96)$ \\
-0.13 & $(-0.91)$ \\
$-0.35^{\star \star}$ & $(-2.18)$ \\
$-0.15^{\star}$ & $(-1.87)$ \\
0.32 & $(1.05)$ \\
$-0.42^{\star \star}$ & $(-2.38)$ \\
0.02 & $(0.11)$ \\
-0.43 & $(-1.40)$ \\
$0.31^{\star \star}$ & $(2.21)$ \\
$-0.45^{\star \star \star}$ & $(-3.34)$ \\
-0.15 & $(-0.45)$ \\
0.02 & $(0.08)$ \\
$1.09^{\star}$ & $(1.89)$ \\
$-0.20^{\star}$ & $(-1.73)$ \\
0.07 & $(0.38)$ \\
0.12 & $(0.78)$ \\
$-0.33^{\star}$ & $(-1.70)$ \\
-0.23 & $(-0.94)$ \\
-0.06 & $(-0.56)$ \\
$-0.19^{\star \star \star}$ & $(-2.87)$ \\
\hline
\end{tabular}


biased in the presence of a confounding event with effects that vary across firms with different leverage ratios. (By contrast, in a panel regression, the effects of random confounding events in each reform will cancel each other out.) Thus, the results need to be interpreted with a grain of salt. For this reason, we prefer to rely on the estimated coefficients aggregated over all reforms rather than focus on the coefficient estimated separately for each individual reform. Assuming that confounding events occur randomly across countries, the aggregated coefficient is more likely to capture the true effect of tax reforms.

As Table 9 shows, the coefficient of LEVERAGE ${ }_{t-1}$ is negative for 18 out of 25 large reforms. Thus, for the majority of reforms, the coefficient is in line with theoretical predictions. Further, the coefficient of LEVERAGE ${ }_{t-1}$ is statistically significant for nine out of the 18 reforms with a negative coefficient. The statistically significant results typically involve countries with a larger number of firms in Worldscope. For example, the median number of observations is 251 for the nine reforms with a negative and significant coefficient, while the median number of observations is 79 for the reforms that exhibit a negative but statistically insignificant coefficient. (We conjecture that the lack of significance might be due to the weak power of tests based on small samples.) The reforms with a negative and significant coefficient of LEVERAGE ${ }_{t-1}$ are Australia (1988), Australia (1993), France (1986), France (1992), Germany (2001), Japan (2000), Portugal (2004), the United Kingdom (1984), and the United States (1988). Additionally, for 7 of the 25 tax cuts in the sample, the coefficient of LEVERAGE L $_{t-1}$ is in conflict with our hypothesis. The coefficient is positive and significant only in two cases (i.e., Italy (2008) and Poland (2004)). Interestingly, both Italy and Poland have "above-median" levels of tax evasion. Thus, overall, when looking at reforms individually, we conclude that the glass of evidence appears to be rather full.

\section{Firms with Positive Earnings}

Our simple discounted cash flow model is applicable to the extent that expected operating cash flows are positive. To simplify the estimation of expected operating cash flows, we rely on the assumption that current operating cash flows provide a good estimate of expected (future) operating cash flows. While this may be reasonable, on average, an obvious problem arises for those firms that have negative current operating cash flows. For those firms, our simple valuation model predicts a negative equity value. We address this issue, to some extent, in Section IV, where we show that tax reforms (and debt tax shields) have a larger impact on value among highly profitable firms as compared with less profitable ones. In this section, we further assess any possible biases in our estimation by excluding firm-years with negative $E_{t} / A_{t-1}$ from the sample. The results are reported in regression 1 of Table 10. The results show that, for this subsample, the impact of tax reforms affecting corporate and personal tax rates on dividend income is greatly mitigated in the presence of leverage. These results are consistent with a tax story.

In regression 1 of Table 10 , it is somewhat surprising to notice that the $E_{t} / A_{t-1} \times$ CORPORATE_TAX_CHANGE interaction is not statistically significant. We, therefore, investigate this issue further. To capture expected cash flows more precisely, we alternatively use a rolling 3-year average of past 
TABLE 10

Other Robustness Tests

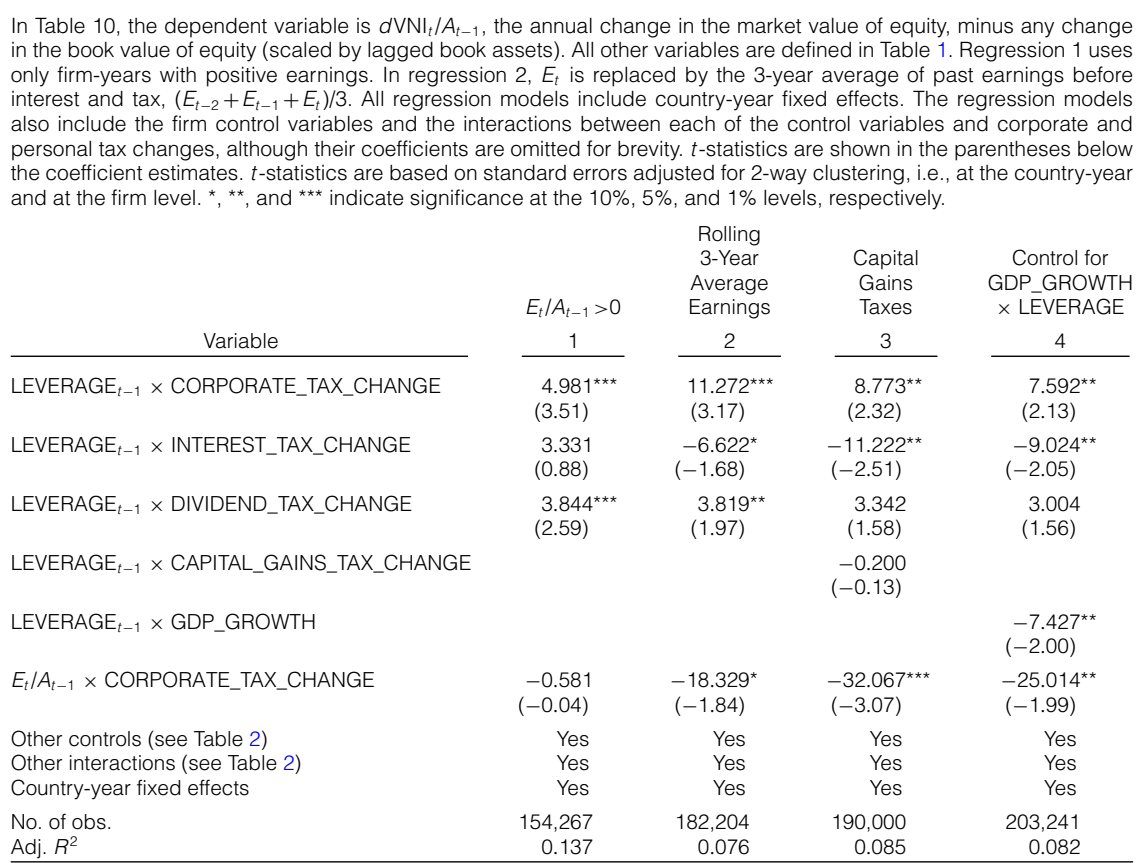

cash flows $\left(\left(E_{t-2}+E_{t-1}+E_{t}\right) / 3\right)$. Those results are reported in regression 2 . With this measure of expected cash flows, the coefficient on the interaction between $\left(\left(\left(E_{t-2}+E_{t-1}+E_{t}\right) / 3\right) / A_{t-1}\right) \times$ CORPORATE_TAX_CHANGE is negative and statistically significant, as one would expect. Importantly, our interaction of interest remains statistically significant.

\section{Capital Gains Taxes}

In our analyses so far, we have not incorporated the taxation of capital gains. Neither the OECD's Tax Database nor the World Bank's World Development Indicators reports data on capital gains taxes. However, Becker, Jacob, and Jacob (2013) compiled capital gains tax rates data for 25 OECD countries for the period of 1990-2008. We verify and supplement those data with news articles from Factiva, the University of Michigan's World Tax Database, the Tax Foundation, internet sources, and other country-specific data sources. Those sources allow us to gather data going back to 1981 for 20 OECD countries and extend the coverage to 28 OECD countries. ${ }^{18}$

We use those data to integrate our earlier analyses with capital gains tax reforms (in addition to reforms affecting personal and corporate income tax rates). As the results in regression 3 of Table 10 show, capital gains tax reforms do not appear to affect the equity value of firms. This could be due to investors being

\footnotetext{
${ }^{18}$ Notably, many countries have a capital gains tax rate of $0 \%$.
} 
able to offset capital gains with capital losses, thus deferring taxation even while selling stocks. Furthermore, in contrast to the other tax reforms, reforms involving capital gains taxes tend to be temporary. Importantly, the tax deductibility of interest at the corporate level remains significant after controlling for this additional type of taxation.

\section{E. Business Cycle}

In this section, we attempt to address the concern that the different change in value experienced by firms with different leverage ratios may not reflect debt tax shields. Rather, it might reflect the different responses of firms, with different degrees of leverage, to macroeconomic shocks that may coincide with tax reforms. This concern is partially mitigated by including the triple interactions of the "cyclical" industry indicator with leverage and tax changes in the main regression model in Table 2. (In that model, we find little evidence that the coefficient of the LEVERAGE LE $_{t-1} \times$ TAX_CHANGE interactions is larger for firms in industries that are expected to be more cyclical.) To further address this concern, we add an interaction between GDP_GROWTH and LEVERAGE ${ }_{t-1}$. GDP_GROWTH is the per capita GDP growth obtained from the World Bank. As the results in regression 4 of Table 10 show, firms with different leverage ratios respond differently to macroeconomic shocks. However, we continue to find evidence consistent with a tax savings story after controlling for other events that could affect equity values through a leverage channel.

\section{F. Other Robustness Tests}

In some additional robustness tests (reported in the Internet Appendix, available at www.jfqa.org), we document that the results are robust to controlling for changes in borrowing and changes in earnings that occur in the year of and 2 years following a reform. The results are also robust to focusing on central government corporate tax rates, while ignoring "sub-central" tax rates such as local taxes. Further, we document that the results are robust to using the lagged market value of assets instead of the lagged book value of assets as a scaling mechanism. Finally, we also verify and confirm the robustness of our results to an alternative measurement of the dependent variable, following Baker and Wurgler (2002).

\section{Conclusions}

Using shocks to tax benefits of debt induced by tax reforms, we estimate the market value of debt tax savings. In line with a simple tax story, we document that, in the time series, the impact of reforms on value differs across firms as a function of leverage. For example, while an increase in corporate tax rates negatively affects equity values, this effect is substantially mitigated in the presence of high leverage.

A battery of other tests corroborates a tax explanation of our results. In particular, the impact of tax reforms differs across firms depending on their tax status; following an increase in corporate tax rates, debt tax shields are more valuable for high corporate taxpayers and more profitable firms. The impact of tax reforms 
also varies across countries in intuitive ways. For example, we find that tax reforms have a lower impact on value in countries with high levels of tax evasion. In those countries, the market value of debt tax savings is (perhaps not surprisingly) also lower. Similarly, reforms have a smaller impact on value in countries where insiders can easily shelter income from taxation by other means (such as stealing).

The results are not driven by unobserved country-level shocks that might equally affect the value of all firms in a given country, which are accounted for through the inclusion of country-year FEs. The results also do not appear to occur through channels other than leverage (such as firm-level growth, changes in (positive net present value) investment opportunities, and/or the discounting of future cash flows). Further, and perhaps most important, they are also robust to narrowing the event window for measuring value changes so as to theoretically filter out events other than the tax reforms.

By using tax reforms as shocks to tax benefits of debt, we are able to provide a clear answer to a core question in the profession of corporate finance: What is the market value of debt tax shields? We find debt tax savings to be highly valued by the market. In particular, our empirical estimates of the tax benefits of debt financing are consistent with the benchmark implied by a traditional approach.

\section{References}

Baker, M., and J. Wurgler. "Market Timing and Capital Structure.” Journal of Finance, 57 (2002), $1-32$.

Bargeron, L.; D. J. Denis; and K. Lehn. "Financing Investment Spikes When the Tax Code Favors Equity: U.S. Firms in the Years Surrounding World War I." Journal of Financial Economics, forthcoming (2018).

Becker, B.; M. Jacob; and M. Jacob. "Payout Taxes and the Allocation of Investment." Journal of Financial Economics, 107 (2013), 1-24.

Bradley, M.; G. Jarrell; and E. H. Kim. "On the Existence of an Optimal Capital Structure: Theory and Evidence." Journal of Finance, 39 (1984), 857-878.

Campello, M. “Taxes and Capital Structure: Do Investors' Taxes Matter? Evidence from the Tax Reform Act of 1986.” Ph.D. Dissertation, University of Illinois at Urbana-Champaign (2001).

Dammon, R. M., and L. W. Senbet. "The Effect of Taxes and Depreciation on Corporate Investment and Financial Leverage." Journal of Finance, 43 (1988), 357-373.

DeAngelo, H., and R. W. Masulis. "Optimal Capital Structure under Corporate and Personal Taxation." Journal of Financial Economics, 8 (1980), 3-29.

Denis, D. J., and I. Osobov. "Why Do Firms Pay Dividends? International Evidence on the Determinants of Dividend Policy.” Journal of Financial Economics, 89 (2008), 62-82.

Desai, M. A.; A. Dyck; and L. Zingales. "Theft and Taxes." Journal of Financial Economics, 84 (2007), 591-623.

Desai, M.; F. Foley; and J. Hines. "A Multinational Perspective on Capital Structure Choice and Internal Capital Markets.” Journal of Finance, 59 (2004), 2451-2487.

Djankov, S.; R. La Porta; F. Lopez-de-Silanes; and A. Shleifer. "The Law and Economics of Self-Dealing." Journal of Financial Economics, 88 (2008), 430-465.

Doidge, C., and A. Dyck. "Taxes and Corporate Policies: Evidence from a Quasi Natural Experiment." Journal of Finance, 70 (2015), 45-89.

Dotan, A., and S. A. Ravid. "On the Interaction of Real and Financial Decisions of the Firm under Uncertainty." Journal of Finance, 40 (1985), 501-517.

Dyreng, S. D.; M. Hanlon; and E. L. Maydew. "Long-Run Corporate Tax Avoidance." Accounting Review, 83 (2008), 61-82.

Faccio, M., and J. Xu. “Taxes and Capital Structure.” Journal of Financial and Quantitative Analysis, 50 (2015), 277-300.

Fama, E. F. “My Life in Finance.” Annual Review of Financial Economics, 3 (2011), 1-15. 
Fama, E. F., and K. R. French. "Taxes, Financing Decisions, and Firm Value.” Journal of Finance, 53 (1998), 819-843.

Fama, E. F., and K. R. French. "Disappearing Dividends: Changing Firm Characteristics or Lower Propensity to Pay?" Journal of Financial Economics, 60 (2001), 3-43.

Fama, E. F., and J. D. MacBeth. "Risk, Return, and Equilibrium: Empirical Tests." Journal of Political Economy, 81 (1973), 607-636.

Fan, J. P. H.; S. Titman; and G. Twite. "An International Comparison of Capital Structure and Debt Maturity Choices." Journal of Financial and Quantitative Analysis, 47 (2012), 23-56.

Givoly, D.; C. Hahn; A. R. Ofer; and O. Sarig. "Taxes and Capital Structure: Evidence from Firms' Response to the Tax Reform Act of 1986." Review of Financial Studies, 52 (1992), 331-355.

Gordon, R. H., and J. K. MacKie-Mason. "Effects of the Tax Reform Act of 1986 on Corporate Financial Policy and Organizational Form." In Do Taxes Matter? The Impact of the Tax Reform Act of 1986, J. Slemrod, ed. Cambridge, MA: MIT Press (1991), 91-131.

Graham, J. R. "How Big Are the Tax Benefits of Debt?" Journal of Finance, 55 (2000), 1901-1941.

Graham, J. R., and M. T. Leary. "A Review of Capital Structure Research and Directions for the Future." Annual Review of Financial Economics, 3 (2011), 309-345.

Green, R. C., and B. Hollifield. "The Personal-Tax Advantages of Equity." Journal of Financial Economics, 67 (2003), 175-216.

Guedhami, O., and J. Pittman. "The Importance of IRS Monitoring to Debt Pricing in Private Firms." Journal of Financial Economics, 90 (2008), 38-58.

Heider, F., and A. Ljungqvist. "As Certain as Debt and Taxes: Estimating the Tax Sensitivity of Leverage from State Tax Changes." Journal of Financial Economics, 118 (2015), 684-712.

Korteweg, A. "The Net Benefits to Leverage." Journal of Finance, 65 (2010), 2137-2170.

Masulis, R. W. "The Effects of Capital Structure Changes on Security Prices: A Study of Exchange Offers." Journal of Financial Economics, 8 (1980), 139-177.

McConnell, J. J., and G. G. Schlarbaum. "Evidence on the Impact of Exchange Offers on Security Prices: The Case of Income Bonds." Journal of Business, 54 (1981), 65-85.

Miller, M. H. "Debt and Taxes." Journal of Finance, 32 (1977), 261-275.

Modigliani, F., and M. H. Miller. "The Cost of Capital, Corporation Finance, and the Theory of Investment." American Economic Review, 48 (1958), 261-297.

Modigliani, F., and M. H. Miller. "Corporate Income Taxes and the Cost of Capital: A Correction." American Economic Review, 56 (1963), 333-391.

Rajan, R., and L. Zingales. "What Do We Know about Capital Structure? Some Evidence from International Data." Journal of Finance, 50 (1995), 1421-1460.

Rosenbaum, P. R., and D. B. Rubin. "The Central Role of the Propensity Score in Observational Studies for Causal Effects." Biometrika, 70 (1983), 41-55.

Twite, G. "Capital Structure Choices and Taxes: Evidence from the Australian Dividend Imputation Tax System." International Review of Finance, 4 (2001), 217-234.

van Binsbergen, J. H.; J. R. Graham; and J. Yang. "The Cost of Debt.” Journal of Finance, 65 (2010), 2089-2136. 